\title{
THE ORBIFOLD CHOW RING OF HYPERTORIC DELIGNE-MUMFORD STACKS
}

\author{
YUNFENG JIANG AND HSIAN-HUA TSENG
}

\begin{abstract}
Hypertoric varieties are determined by hyperplane arrangements. In this paper, we use stacky hyperplane arrangements to define the notion of hypertoric Deligne-Mumford stacks. Their orbifold Chow rings are computed. As an application, some examples related to crepant resolutions are discussed.
\end{abstract}

\section{INTRODUCTION}

Hypertoric varieties (cf. $[\mathrm{BD},[\mathrm{P})$ ) are the hyperkähler analogue of Kähler toric varieties. The algebraic construction of hypertoric varieties was given by Hausel and Sturmfels [HS]. Modelling on their construction, in this paper we construct hypertoric DM stacks and study their orbifold Chow rings.

According to $[\mathrm{BD}$, the topology of hypertoric varieties is determined by hyperplane arrangements. In this paper we define stacky hyperplane arrangements from which we define hypertoric DM stacks.

Let $N$ be a finitely generated abelian group of rank $d$ and $N \rightarrow \bar{N}$ the natural projection modulo torsion. Let $\beta: \mathbb{Z}^{m} \rightarrow N$ be a homomorphism determined by a collection of nontorsion integral vectors $\left\{b_{1}, \cdots, b_{m}\right\} \subseteq N$. We require that $\beta$ has finite cokernel. The Gale dual of $\beta$ is denoted by $\beta^{\vee}:\left(\mathbb{Z}^{m}\right)^{*} \rightarrow D G(\beta)$. A generic element $\theta$ in $D G(\beta)$ and the vectors $\left\{\bar{b}_{1}, \cdots, \bar{b}_{m}\right\}$ determine a hyperplane arrangement $\mathcal{H}=\left(H_{1}, \cdots, H_{m}\right)$ in $N_{\mathbb{R}}^{*}$. We call $\mathcal{A}:=(N, \beta, \theta)$ a stacky hyperplane arrangement.

For $\beta: \mathbb{Z}^{m} \rightarrow N$ in $\mathcal{A}$, we consider the Lawrence lifting $\beta_{L}: \mathbb{Z}^{m} \oplus \mathbb{Z}^{m} \rightarrow N_{L}$ of $\beta$ where $N_{L}$ is a finitely generated abelian group with rank $m+d$. The map $\beta_{L}$ is given by vectors $\left\{b_{L, 1}, \cdots, b_{L, m}, b_{L, 1}^{\prime}, \cdots, b_{L, m}^{\prime}\right\} \subseteq N_{L}$. The generic element $\theta$ determines a Lawrence simplicial fan $\Sigma_{\theta}$ in $\bar{N}_{L}$. We call $\boldsymbol{\Sigma}_{\theta}=\left(N_{L}, \Sigma_{\theta}, \beta_{L}\right)$ a Lawrence stacky fan and $\mathcal{X}\left(\boldsymbol{\Sigma}_{\theta}\right)$ the Lawrence toric DM stack. The hypertoric DM stack $\mathcal{M}(\mathcal{A})$ associated to $\mathcal{A}$ is defined as a quotient stack which is a closed substack of the Lawrence toric DM stack $\mathcal{X}\left(\boldsymbol{\Sigma}_{\theta}\right)$, generalizing the construction of [HS].

The stacky hyperplane arrangement $\mathcal{A}$ also determines an extended stacky fan $\boldsymbol{\Sigma}=(N, \Sigma, \beta)$ introduced in [Jiang]. Here $\Sigma$ is the normal fan of the bounded polytope $\boldsymbol{\Gamma}$ of the hyperplane arrangement $\mathcal{H}$. The toric DM stack $\mathcal{X}(\boldsymbol{\Sigma})$ defined in [Jiang] is the associated toric DM stack of $\mathcal{M}(\mathcal{A})$.

To the map $\beta$ we associate a multi-fan $\Delta_{\beta}$ in the sense of [HM], which consists of cones generated by linearly independent subsets $\left\{\bar{b}_{i_{1}}, \cdots, \bar{b}_{i_{k}}\right\}$ in $\bar{N}$ for $\left\{i_{1}, \cdots, i_{k}\right\} \subset\{1, \cdots, m\}$,

Date: October 16, 2018. 
see Section 4. We assume that the $\operatorname{supp}\left(\Delta_{\beta}\right)=\bar{N}$. We prove that each top dimensional cone in $\Delta_{\beta}$ gives a local chart for the hypertoric DM stack $\mathcal{M}(\mathcal{A})$. We define a set $\operatorname{Box}\left(\Delta_{\beta}\right)$ consisting of all pairs $(v, \sigma)$, where $\sigma$ is a cone in the multi-fan $\Delta_{\beta}, v \in N$ such that $\bar{v}=\sum_{\rho_{i} \subset \sigma} \alpha_{i} \bar{b}_{i}$ for $0<\alpha_{i}<1$. For $(v, \sigma) \in \operatorname{Box}\left(\Delta_{\beta}\right)$ we consider a closed substack of $\mathcal{M}(\mathcal{A})$ given by the quotient stacky hyperplane arrangement $\mathcal{A}(\sigma)$. The inertia stack of $\mathcal{M}(\mathcal{A})$ is the disjoint union of all such closed substacks, see Section 4 .

We now describe the orbifold Chow ring of $\mathcal{M}(\mathcal{A})$. The multi-fan $\Delta_{\beta}$ naturally gives a "matroid" $M_{\beta}$. The vertex set is $\{1, \cdots, m\}$, and the faces are the subsets $\left\{i_{1}, \cdots, i_{k}\right\} \subseteq$ $\{1, \cdots, m\}$ such that $\left\{\bar{b}_{i_{1}}, \cdots, \bar{b}_{i_{k}}\right\}$ are linearly independent in $\bar{N}$. Note that the faces of $M_{\beta}$ are the cones in $\Delta_{\beta}$. According to [HS], the ordinary cohomology ring of the hypertoric variety corresponding to the hyperplane arrangement $\mathcal{H}$ is isomorphic to the "Stanley-Reisner" ring of the matroid $M_{\beta}$. Our result shows that the orbifold Chow ring of hypertoric DM stacks is a generalization of the Stanley-Reisner ring of the matroid $M_{\beta}$ to the multi-fan $\Delta_{\beta}$. Let $N^{\Delta_{\beta}}$ denote all the pairs $(c, \sigma)$, where $c \in N, \sigma$ is a cone in $\Delta_{\beta}$ such that $\bar{c}=\sum_{\rho_{i} \subseteq \sigma} a_{i} \bar{b}_{i}$ and $a_{i}>0$ are rational numbers. Then $N^{\Delta_{\beta}}$ gives rise to a group ring

$$
\mathbb{Q}\left[\Delta_{\beta}\right]=\bigoplus_{(c, \sigma) \in N^{\Delta_{\beta}}} \mathbb{Q} \cdot y^{(c, \sigma)},
$$

where $y$ is a formal variable. For any $(c, \sigma) \in N^{\Delta_{\beta}}$, there exists a unique element $(v, \tau) \in$ $\operatorname{Box}\left(\Delta_{\beta}\right)$ such that $\tau \subset \sigma$ and $c=v+\sum_{\rho_{i} \subseteq \sigma} m_{i} b_{i}$, where $m_{i}$ are nonnegative integers. We call $(v, \tau)$ the fractional part of $(c, \sigma)$. For $(c, \sigma)$ we define the ceiling function $\lceil c\rceil_{\sigma}$ by $\lceil c\rceil_{\sigma}=\sum_{\rho_{i} \subseteq \tau} b_{i}+\sum_{\rho_{i} \subseteq \sigma} m_{i} b_{i}$. Note that if $\bar{v}=0,\lceil c\rceil_{\sigma}=\sum_{\rho_{i} \subseteq \sigma} m_{i} b_{i}$. For two pairs $\left(c_{1}, \sigma_{1}\right)$, $\left(c_{2}, \sigma_{2}\right)$, if $\sigma_{1} \cup \sigma_{2}$ is a cone in $\Delta_{\beta}$, define $\epsilon\left(c_{1}, c_{2}\right):=\left\lceil c_{1}\right\rceil_{\sigma_{1}}+\left\lceil c_{2}\right\rceil_{\sigma_{2}}-\left\lceil c_{1}+c_{2}\right\rceil_{\sigma_{1} \cup \sigma_{2}}$. Let $\sigma_{\epsilon} \subseteq \sigma_{1} \cup \sigma_{2}$ be the minimal cone in $\Delta_{\beta}$ containing $\epsilon\left(c_{1}, c_{2}\right)$ so that $\left(\epsilon\left(c_{1}, c_{2}\right), \sigma_{\epsilon}\right) \in N^{\Delta_{\beta}}$. We define the grading on $\mathbb{Q}\left[\Delta_{\beta}\right]$ as follows. For any $(c, \sigma)$, write $c=v+\sum_{\rho_{i} \subseteq \sigma} m_{i} b_{i}$, then

$$
\operatorname{deg}\left(y^{(c, \sigma)}\right):=|\tau|+\sum_{\rho_{i} \subseteq \sigma} m_{i}
$$

where $|\tau|$ is the dimension of $\tau$. Note that we have integer grading due to the fact that $\mathcal{M}(\mathcal{A})$ is hyperkähler. By abuse of notation, we write $y^{\left(b_{i}, \rho_{i}\right)}$ as $y^{b_{i}}$. The multiplication is defined by

$$
y^{\left(c_{1}, \sigma_{1}\right)} \cdot y^{\left(c_{2}, \sigma_{2}\right)}:= \begin{cases}(-1)^{\left|\sigma_{\epsilon}\right|} y^{\left(c_{1}+c_{2}+\epsilon\left(c_{1}, c_{2}\right), \sigma_{1} \cup \sigma_{2}\right)} & \text { if } \sigma_{1} \cup \sigma_{2} \text { is a cone in } \Delta_{\beta} \\ 0 & \text { otherwise }\end{cases}
$$

Using the property of ceiling functions we check that the multiplication is commutative and associative. So $\mathbb{Q}\left[\Delta_{\beta}\right]$ is a unital associative commutative ring. Let $\operatorname{Cir}\left(\Delta_{\beta}\right)$ be the ideal in $\mathbb{Q}\left[\Delta_{\beta}\right]$ generated by the elements:

$$
\sum_{i=1}^{m} e\left(b_{i}\right) y^{b_{i}}, \quad e \in N^{*}
$$

Let $A_{\text {orb }}^{*}(\mathcal{M}(\mathcal{A}))$ be the orbifold Chow ring of the hypertoric DM stack $\mathcal{M}(\mathcal{A})$. We have the following Theorem: 
Theorem 1.1. Let $\mathcal{M}(\mathcal{A})$ be the hypertoric DM stack associated to the stacky hyperplane arrangement $\mathcal{A}$. Then there is an isomorphism of graded $\mathbb{Q}$-algebras:

$$
A_{\text {orb }}^{*}(\mathcal{M}(\mathcal{A})) \cong \frac{\mathbb{Q}\left[\Delta_{\beta}\right]}{\operatorname{Cir}\left(\Delta_{\beta}\right)}
$$

The orbifold Chow ring of the hypertoric DM stack $\mathcal{M}(\mathcal{A})$ is independent of the generic element $\theta$. It only depends on the map $\beta$.

Theorem 1.1 is proven by a direct approach. The inertia stack of a hypertoric DM stack $\mathcal{M}(\mathcal{A})$ is the disjoint union of closed substacks $\mathcal{M}(\mathcal{A}(\sigma))$ for all $(v, \sigma) \in \operatorname{Box}\left(\Delta_{\beta}\right)$. To determine the ring structure, we identify the 3 -twisted sectors as closed substacks of $\mathcal{M}(\mathcal{A})$ indexed by triples $\left(\left(v_{1}, \sigma_{1}\right),\left(v_{2}, \sigma_{2}\right),\left(v_{3}, \sigma_{3}\right)\right)$ in $\operatorname{Box}\left(\Delta_{\beta}\right)^{3}$ such that $v_{1}+v_{2}+v_{3} \in N$ is a integral linear combination of $b_{i}$ 's. We then determine the obstruction bundle over any 3 twisted sector and prove that the orbifold cup product is the same as the product of the ring $\mathbb{Q}\left[\Delta_{\beta}\right]$ described above.

The multi-fan $\Delta_{\beta}$ is equal to the simplicial fan $\Sigma$ in $\Sigma$ induced from the stacky hyperplane arrangement $\mathcal{A}$ if and only if $\mathcal{H}$ has $n$ hyperplanes $\left\{H_{1}, \cdots, H_{n}\right\}$ whose normal polytope is a product of simplices. So in this case $\Sigma$ is a stacky fan and the simplicial fan $\Sigma$ is a product of normal fans of simplices, the toric variety $X(\Sigma)$ is a product of weighted projective spaces. Then by $[\mathrm{BD}]$ the associated hypertoric variety is the cotangent bundle of the toric variety $X(\Sigma)$. So $\mathcal{M}(\mathcal{A}) \simeq T^{*} \mathcal{X}(\Sigma)$, the cotangent bundle of the toric DM stack $\mathcal{X}(\Sigma)$. The ring $\mathbb{Q}\left[\Delta_{\beta}\right]$ coincides (as vector spaces) with the deformed ring $\mathbb{Q}[N]^{\boldsymbol{\Sigma}}$ as defined in $[$ BCS].

Corollary 1.2. Let $\boldsymbol{\Sigma}$ be as above. Then there is an isomorphism of $\mathbb{Q}$-vector spaces

$$
A_{\text {orb }}^{*}(\mathcal{M}(\mathcal{A})) \simeq A_{\text {orb }}^{*}(\mathcal{X}(\Sigma))
$$

Here is an example which shows that the orbifold Chow ring of $\mathcal{M}(\mathcal{A})$ is not isomorphic as a ring to the orbifold Chow ring of the associated toric DM stack $\mathcal{X}(\boldsymbol{\Sigma})$. Consider the weighted projective stack $\mathbb{P}(1,2)$ which is a toric DM stack with stacky fan $\Sigma=(N, \Sigma, \beta)$, where $N=\mathbb{Z}, \beta: \mathbb{Z}^{2} \rightarrow N$ is given by the vectors $b_{1}=(1), b_{2}=(-2)$ and $\Sigma$ is the simplicial fan in the lattice $N$ consisting cones $\rho_{1}$ and $\rho_{2}$ generated by $b_{1}=(1)$ and $b_{2}=(-2)$ respectively. The Gale dual map $\beta^{\vee}: \mathbb{Z}^{2} \rightarrow \mathbb{Z}$ is given by the matrix (2). Choosing generic element $\theta=(1) \in \mathbb{Z}$, we get a stacky hyperplane arrangement $\mathcal{A}=(N, \beta, \theta)$. The hypertoric DM stack $\mathcal{M}(\mathcal{A})$ is the cotangent bundle $T^{*} \mathbb{P}(1,2)$ whose core is the toric DM stack $\mathbb{P}(1,2)$. Both $\mathbb{Q}\left[\Delta_{\beta}\right]$ and $\mathbb{Q}[N]^{\Sigma}$ are generated by $y^{b_{1}}, y^{b_{2}}$, and $y^{\left(\frac{1}{2} b_{2}, \rho_{2}\right)}$. According to Theorem 1.1 and the main theorem in [BCS], their orbifold Chow rings are given as follows:

$$
\begin{gathered}
A_{\text {orb }}^{*}(\mathcal{X}(\boldsymbol{\Sigma}) ; \mathbb{Q}) \cong \frac{\mathbb{Q}\left[x_{1}, x_{2}, v\right]}{\left(x_{1}-2 x_{2}, v^{2}-x_{2}, v x_{1}, x_{1} x_{2}\right)} \cong \frac{\mathbb{Q}[v]}{\left(v^{3}\right)}, \\
A_{\text {orb }}^{*}(\mathcal{M}(\mathcal{A}) ; \mathbb{Q}) \cong \frac{\mathbb{Q}\left[x_{1}, x_{2}, v\right]}{\left(x_{1}-2 x_{2}, x_{1} x_{2}, v x_{1}, v^{2}\right)} \cong \frac{\mathbb{Q}\left[x_{2}, v\right]}{\left(x_{2}^{2}, v x_{2}, v^{2}\right)} .
\end{gathered}
$$

It is easy to see that these two rings are not isomorphic. Thus the orbifold Chow ring of a hypertoric DM stack is not necessarily isomorphic to the orbifold Chow ring of its core. (However, their Chow rings are isomorphic, see Theorem 1.1 of [HS].) This also proves that the orbifold Chow ring has no homotopy invariance property. We remark that the core of a general hypertoric DM stack can be singular, it is not clear how to define orbifold Chow 
ring. But in the case of a cotangent bundle over weighted projective space, the core is the weighted projective space and the orbifold Chow ring is well-defined. On the other hand, the orbifold Chow ring of a Lawrence toric DM stack is isomorphic to its associated hypertoric DM stack, see [JT].

Computations of orbifold cohomology rings of hypertoric orbifolds in symplectic geometry have been pursued in $[\mathrm{GH}]$.

This paper is organized as follows. In Section 2 we discuss the relation between stacky hyperplane arrangements and extended stacky fans. We define hypertoric DM stack $\mathcal{M}(\mathcal{A})$ associated to the stacky hyperplane arrangement $\mathcal{A}$. In Section 3 we discuss the properties of hypertoric DM stacks. In Section 4 we determine closed substacks of a hypertoric DM stack. This yields a description of its inertia stacks. We prove Theorem 1.1 in Section 5, and in Section [6] we give some examples.

Conventions. In this paper we work entirely algebraically over the field of complex numbers. Chow rings and orbifold Chow rings are taken with rational coefficients. By an orbifold we mean a smooth Deligne-Mumford stack with trivial generic stabilizer. We refer to [BCS] for the construction of Gale dual $\beta^{\vee}: \mathbb{Z}^{m} \rightarrow D G(\beta)$ from $\beta: \mathbb{Z}^{m} \rightarrow N$. We denote by $N \rightarrow \bar{N}$ the natural map modulo torsion. For cones $\sigma_{1}, \sigma_{2}$ in $\mathbb{R}^{d}$, we use $\sigma_{1} \cup \sigma_{2}$ to represent the set of union of the generators of $\sigma_{1}$ and $\sigma_{2}$.

Acknowledgments. We would like to thank the referee for the nice comments of Remark 2.4 and Kai Behrend, Megumi Harada, Nicholas Proudfoot for valuable discussions.

\section{The Hypertoric DM Stacks}

In this section we define hypertoric Deligne-Mumford stacks, mimicking the construction of hypertoric varieties in [HS].

Stacky hyperplane arrangements. We introduce stacky hyperplane arrangements. We explain how a stacky hyperplane arrangement gives extended stacky fans.

Let $N$ be a finitely generated abelian group and $\beta: \mathbb{Z}^{m} \rightarrow N$ a map given by nontorsion integral vectors $\left\{b_{1}, \ldots, b_{m}\right\}$. We have the following exact sequences:

$$
\begin{aligned}
& 0 \longrightarrow D G(\beta)^{*} \stackrel{\left(\beta^{\vee}\right)^{*}}{\longrightarrow} \mathbb{Z}^{m} \stackrel{\beta}{\longrightarrow} N \longrightarrow \operatorname{Coker}(\beta) \longrightarrow 0, \\
& 0 \longrightarrow N^{*} \longrightarrow \mathbb{Z}^{m} \stackrel{\beta^{\vee}}{\longrightarrow} D G(\beta) \longrightarrow \operatorname{Coker}\left(\beta^{\vee}\right) \longrightarrow 0,
\end{aligned}
$$

where $\beta^{\vee}$ is the Gale dual of $\beta$ (see [BCS]). The map $\beta^{\vee}$ is given by the integral vectors $\left\{a_{1}, \cdots, a_{m}\right\} \subseteq D G(\beta)$. Choose a generic element $\theta \in D G(\beta)$ which lies in the inage of $\beta^{\vee}$ and let $\psi:=\left(r_{1}, \cdots, r_{m}\right)$ be a lifting of $\theta$ in $\mathbb{Z}^{m}$ such that $\theta=-\beta^{\vee} \psi$. Note that $\theta$ is generic if and only if it is not in any hyperplane of the configuration determined by $\beta^{\vee}$ in $D G(\beta)_{\mathbb{R}}$. Let $M=N^{*}$ be the dual of $N$ and $M_{\mathbb{R}}=M \otimes_{\mathbb{Z}} \mathbb{R}$, then $M_{\mathbb{R}}$ is a $d$-dimensional $\mathbb{R}$-vector space. Associated to $\theta$ there is a hyperplane arrangement $\mathcal{H}=\left\{H_{1}, \cdots, H_{m}\right\}$ in $M_{\mathbb{R}}$ defined by $H_{i}$ the hyperplane

$$
H_{i}:=\left\{v \in M_{\mathbb{R}} \mid<b_{i}, v>+r_{i}=0\right\} \subset M_{\mathbb{R}}
$$


So (2.3) determines hyperplane arrangements in $M_{\mathbb{R}}$, up to translation induced by the choice of the lifting $\psi:=\left(r_{1}, \cdots, r_{m}\right)$.

Definition 2.1. We call $\mathcal{A}:=(N, \beta, \theta)$ a stacky hyperplane arrangement.

It is well-known that hyperplane arrangements determine the topology of hypertoric varieties [BD]. Let

$$
\boldsymbol{\Gamma}=\bigcap_{i=1}^{m} F_{i}, \text { where } F_{i}=\left\{v \in M_{\mathbb{R}} \mid<b_{i}, v>+r_{i} \geq 0\right\} .
$$

Let $\Sigma$ be the normal fan of $\boldsymbol{\Gamma}$ in $M_{\mathbb{R}}=\mathbb{R}^{d}$ with one dimensional rays generated by $\bar{b}_{1}, \cdots, \bar{b}_{n}$. By reordering, we may assume that $H_{1}, \cdots, H_{n}$ are the hyperplanes that bound the polytope $\boldsymbol{\Gamma}$, and $H_{n+1}, \cdots, H_{m}$ are the other hyperplanes. Then we have an extended stacky fan $\boldsymbol{\Sigma}=$ $(N, \Sigma, \beta)$ defined in Jiang, where $\beta: \mathbb{Z}^{m} \rightarrow N$ is given by $\left\{b_{1}, \cdots, b_{n}, b_{n+1}, \cdots, b_{m}\right\} \subset N$, and $\left\{b_{n+1}, \cdots, b_{m}\right\}$ are the extra data.

By Jiang, the extended stacky fan $\boldsymbol{\Sigma}$ determines a toric Deligne-Mumford stack $\mathcal{X}(\boldsymbol{\Sigma})$. It is the same stack as in [BCS]. Its coarse moduli space is the toric variety corresponding to the normal fan of $\boldsymbol{\Gamma}$. According to $[\mathrm{BD}$, a hyperplane arrangement $\mathcal{H}$ is simple if the codimension of the nonempty intersection of any $l$ hyperplanes is $l$. A hypertoric variety is the coarse moduli space of an orbifold if the corresponding hyperplane arrangement is simple.

Example 2.2. Let $\mathcal{H}=\left\{H_{1}, H_{2}, H_{3}, H_{4}\right\}$, see Figure 1. The polytope $\boldsymbol{\Gamma}$ of the hyperplane arrangement is the shaded triangle whose toric variety is the projective plane. The extended stacky fan is given by the fan of the projective plane $\mathbb{P}^{2}$ and an extra ray $(0,1)$.
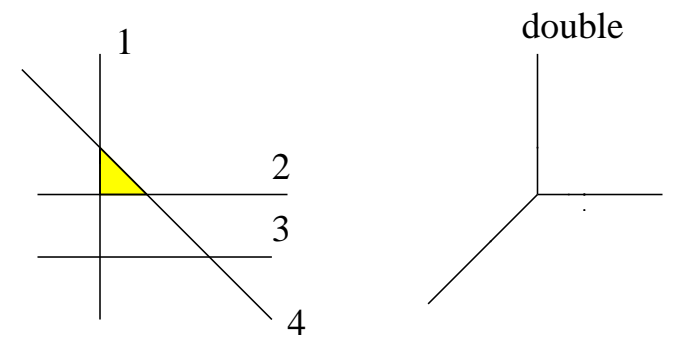

Figure 1: The correspondence of the hyperplane arrangement and an extended stacky fan

Remark 2.3. If for a generic element $\theta \in D G(\beta)$ the hyperplane arrangement $\mathcal{H}$ bounds a polytope whose normal fan is $\Sigma$, then $\Sigma=(N, \Sigma, \beta)$ is a stacky fan defined in [BCS].

Lawrence toric DM stacks. Consider the Gale dual map $\beta^{\vee}: \mathbb{Z}^{m} \rightarrow D G(\beta)$ in (2.2). We denote the Gale dual map of

$$
\mathbb{Z}^{m} \oplus \mathbb{Z}^{m} \stackrel{\left(\beta^{\vee},-\beta^{\vee}\right)}{\longrightarrow} D G(\beta)
$$

by

$$
\beta_{L}: \mathbb{Z}^{2 m} \rightarrow N_{L}
$$

where $\bar{N}_{L}$ is a lattice of dimension $2 m-(m-d)$. The map $\beta_{L}$ is given by the integral vectors $\left\{b_{L, 1}, \cdots, b_{L, m}, b_{L, 1}^{\prime}, \cdots, b_{L, m}^{\prime}\right\}$ and $\beta_{L}$ is called the Lawrence lifting of $\beta$. 
Remark 2.4. Consider the following commutative diagram for which the rows are exact:

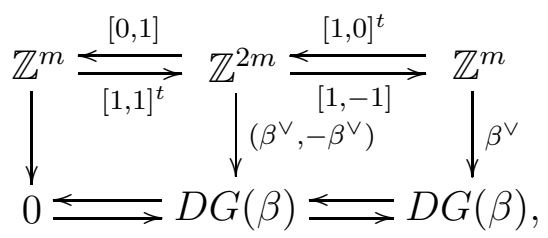

where $[1,1]^{t}$ represents the transpose of the matrix. Taking Gale dual to the above diagram yields the following commutative diagram:

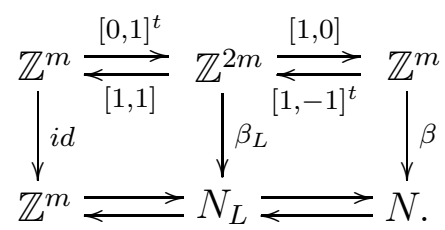

So from the functoriality of Gale dual we have $N_{L} \cong N \oplus \mathbb{Z}^{m}$. Since $\left(\beta^{\vee},-\beta^{\vee}\right)=\beta^{\vee} \oplus 0$, we get that $\beta_{L}=\beta \oplus i d$. So $\left\{b_{L, 1}, \cdots, b_{L, m}, b_{L, 1}^{\prime}, \cdots, b_{L, m}^{\prime}\right\}$ are the vectors

$$
\left\{\left(b_{1}, e_{1}\right), \cdots,\left(b_{m}, e_{m}\right),\left(0, e_{1}\right), \cdots,\left(0, e_{m}\right)\right\},
$$

where $\left\{e_{i}\right\}$ are the standard bases of $\mathbb{Z}^{m}$.

Given the generic element $\theta$, let $\bar{\theta}$ be the natural image of $\theta$ under the projection $D G(\beta) \rightarrow$ $\overline{D G(\beta)}$. Then the map $\bar{\beta}^{\vee}: \mathbb{Z}^{m} \rightarrow \overline{D G(\beta)}$ is given by $\bar{\beta}^{\vee}=\left(\bar{a}_{1}, \cdots, \bar{a}_{m}\right)$. For any basis of $\overline{D G(\beta)}$ of the form $C=\left\{\bar{a}_{i_{1}}, \cdots, \bar{a}_{i_{m-d}}\right\}$, there exist unique $\lambda_{1}, \cdots, \lambda_{m-d}$ such that

$$
\bar{a}_{i_{1}} \lambda_{1}+\cdots+\bar{a}_{i_{m-d}} \lambda_{m-d}=\bar{\theta} .
$$

Let $\mathbb{C}\left[z_{1}, \cdots, z_{m}, w_{1}, \cdots, w_{m}\right]$ be the coordinate ring of $\mathbb{C}^{2 m}$. Let

$$
\sigma(C, \theta)=\left\{\bar{b}_{L, i_{j}} \mid \lambda_{j}>0\right\} \sqcup\left\{\bar{b}_{L, i_{j}}^{\prime} \mid \lambda_{j}<0\right\} \quad \text { and } \quad C(\theta)=\left\{z_{i_{j}} \mid \lambda_{j}>0\right\} \sqcup\left\{w_{i_{j}} \mid \lambda_{j}<0\right\} .
$$

We put

$$
\left.\mathcal{I}_{\theta}:=\left\langle\prod C(\theta)\right| C \text { is a basis of } \overline{D G(\beta)}\right\rangle,
$$

and

$$
\Sigma_{\theta}:=\{\bar{\sigma}(C, \theta): C \text { is a basis of } \overline{D G(\beta)}\},
$$

where $\bar{\sigma}(C, \theta)=\left\{\bar{b}_{L, 1}, \cdots, \bar{b}_{L, m}, \bar{b}_{L, 1}^{\prime}, \cdots, \bar{b}_{L, m}^{\prime}\right\} \backslash \sigma(C, \theta)$ is the complement of $\sigma(C, \theta)$ and corresponds to a maximal cone in $\Sigma_{\theta}$. From [HS], $\Sigma_{\theta}$ is the fan of a Lawrence toric variety $X\left(\Sigma_{\theta}\right)$ corresponding to $\theta$ in the lattice $\bar{N}_{L}$, and $\mathcal{I}_{\theta}$ is the irrelevant ideal. The construction above establishes the following

Proposition 2.5. A stacky hyperplane arrangement $\mathcal{A}=(N, \beta, \theta)$ also gives a stacky fan $\Sigma_{\theta}=\left(N_{L}, \Sigma_{\theta}, \beta_{L}\right)$ which is called a Lawrence stacky fan.

Proof. From Proposition 4.3 in [HS], $\Sigma_{\theta}$ is a simplicial fan in $\bar{N}_{L}$. The rays $\rho_{L, i}, \rho_{L, i}^{\prime}$ are generated by $\bar{b}_{L, i}, \bar{b}_{L, i}^{\prime}$. The map $\beta_{L}$ is the map (2.4) given by $\left\{b_{L, 1}, \cdots, b_{L, m}, b_{L, 1}^{\prime}, \cdots, b_{L, m}^{\prime}\right\}$. So by [BCS], $\Sigma_{\theta}=\left(N_{L}, \Sigma_{\theta}, \beta_{L}\right)$ is a stacky fan.

Definition 2.6. The toric DM stack $\mathcal{X}\left(\boldsymbol{\Sigma}_{\theta}\right)$ is called the Lawrence toric DM stack. 
For the map $\beta_{L}^{\vee}: \mathbb{Z}^{m} \oplus \mathbb{Z}^{m} \rightarrow D G(\beta)$ given by $\left(\beta^{\vee},-\beta^{\vee}\right)$, there is an exact sequence

$$
0 \longrightarrow N_{L}^{*} \longrightarrow \mathbb{Z}^{2 m} \stackrel{\beta_{L}^{\vee}}{\longrightarrow} D G(\beta) \longrightarrow \operatorname{Coker}\left(\beta_{L}^{\vee}\right) \longrightarrow 0 .
$$

Applying $\operatorname{Hom}_{\mathbb{Z}}\left(-, \mathbb{C}^{\times}\right)$to (2.7) yields

$$
1 \longrightarrow \mu \longrightarrow G \stackrel{\alpha^{L}}{\longrightarrow}\left(\mathbb{C}^{\times}\right)^{2 m} \longrightarrow T_{L} \longrightarrow 1
$$

where $\mu:=\operatorname{Hom}_{\mathbb{Z}}\left(\operatorname{Coker}\left(\beta_{L}^{\vee}\right), \mathbb{C}^{\times}\right)$and $T_{L}$ is the torus of dimension $m+d$. From [BCS] and Proposition 2.5, the toric DM stack $\mathcal{X}\left(\boldsymbol{\Sigma}_{\theta}\right)$ is the quotient stack $\left[\left(\mathbb{C}^{2 m} \backslash V\left(\mathcal{I}_{\theta}\right)\right) / G\right]$, where $G$ acts on $\mathbb{C}^{2 m} \backslash V\left(\mathcal{I}_{\theta}\right)$ through the map $\alpha^{L}$ in (2.8).

Hypertoric DM stacks. Define an ideal in $\mathbb{C}[z, w]$ by:

$$
I_{\beta^{\vee}}:=\left\langle\sum_{i=1}^{m}\left(\beta^{\vee}\right)^{*}(x)_{i} z_{i} w_{i} \mid x \in D G(\beta)^{*}\right\rangle,
$$

where $\left(\beta^{\vee}\right)^{*}$ is the map in (2.1) and $\left(\beta^{\vee}\right)^{*}(x)_{i}$ is the $i$-th component of the vector $\left(\beta^{\vee}\right)^{*}(x)$.

According to Section 6 in [HS], $I_{\beta} \vee$ is a prime ideal. Let $Y$ be the closed subvariety of $\mathbb{C}^{2 m} \backslash V\left(\mathcal{I}_{\theta}\right)$ determined by the ideal (2.9). Since $\left(\mathbb{C}^{\times}\right)^{2 m}$ acts on $Y$ naturally and the group $G$ acts on $Y$ through the map $\alpha^{L}$, we have the quotient stack $[Y / G]$. Since $Y \subseteq \mathbb{C}^{2 m} \backslash V\left(\mathcal{I}_{\theta}\right)$ is a closed subvariety, the quotient stack $[Y / G]$ is a closed substack of $\mathcal{X}\left(\boldsymbol{\Sigma}_{\theta}\right)$, and is DeligneMumford.

Definition 2.7. The hypertoric Deligne-Mumford stack $\mathcal{M}(\mathcal{A})$ associated to the stacky hyperplane arrangement $\mathcal{A}$ is defined to be the quotient stack $[Y / G]$.

Example 2.8. Let $N=\mathbb{Z} \oplus \mathbb{Z}_{2}$, $\Sigma$ the fan of projective line $\mathbb{P}^{1}$, and $\beta: \mathbb{Z}^{3} \rightarrow N$ given by $\left\{b_{1}=(1,0), b_{2}=(-1,1), b_{3}=(1,0)\right\}$. Then the Gale dual $\beta^{\vee}: \mathbb{Z}^{3} \rightarrow \mathbb{Z}^{2}$ is given by the matrix $\left[\begin{array}{lll}1 & 0 & 1 \\ 2 & 2 & 0\end{array}\right]$. Choose a generic element $\theta=(1,1)$ in $\mathbb{Z}^{2}$ which determines the fan $\Sigma$. The stacky hyperplane arrangement is $\mathcal{A}=(N, \beta, \theta), G=\left(\mathbb{C}^{\times}\right)^{2}$ and $Y$ is the subvariety of $\operatorname{Spec}\left(\mathbb{C}\left[z_{1}, z_{2}, z_{3}, w_{1}, w_{2}, w_{3}\right]\right)$ determined by the ideal $I_{\beta^{\vee}}=\left(z_{1} w_{1}+z_{3} w_{3}, 2 z_{1} w_{1}+2 z_{2} w_{2}\right)$. Then by $[\mathrm{HS}]$, the coarse moduli space is the crepant resolution of the Gorenstein orbifold $\left[\mathbb{C}^{2} / \mathbb{Z}_{3}\right]$, see Figure 3. The corresponding hyperplane arrangement $\mathcal{H}$ consists of three distinct points on the real line $\mathbb{R}^{1}$, and the bounded polyhedron is two segments intersecting at one point. So the core of the hypertoric variety is two $\mathbb{P}^{1}$ intersecting at one point. The hypertoric DM stack $\mathcal{M}(\mathcal{A})$ is a nontrivial $\mu_{2}$-gerbe over the crepant resolution according to the action given by the inverse of the above matrix. If we change $b_{2}$ to $(-1,0)$, we will see an example in Section 4 that the hypertoric DM stack is a trivial $\mu_{2}$-gerbe over the crepant resolution.

\section{Properties of Hypertoric DM Stacks}

The coarse moduli space. Each Deligne-Mumford stack has an underlying coarse moduli space. In this section we prove that the coarse moduli space of $\mathcal{M}(\mathcal{A})$ is the underlying hypertoric variety.

Consider again the map $\beta^{\vee}: \mathbb{Z}^{m} \rightarrow D G(\beta)$ in (2.2), which is given by the vectors $\left(a_{1}, \cdots, a_{m}\right)$. As in Section 2 , let $\bar{\theta}$ be the natural image of $\theta$ under the projection $D G(\beta) \rightarrow$ 
$\overline{D G(\beta)}$. Then the map $\bar{\beta}^{\vee}: \mathbb{Z}^{m} \rightarrow \overline{D G(\beta)}$ is given by $\bar{\beta}^{\vee}=\left(\bar{a}_{1}, \cdots, \bar{a}_{m}\right)$. From the map $\bar{\beta}^{\vee}$ we get the simplicial fan $\Sigma_{\theta}$ in $(2.6)$. By [BCS], the toric variety $X\left(\Sigma_{\theta}\right)$, which is the geometric quotient $\left(\mathbb{C}^{2 m}-V\left(\mathcal{I}_{\theta}\right)\right) / G$, is the coarse moduli space of the Lawrence toric DM stack $\mathcal{X}\left(\Sigma_{\theta}\right)$. The toric variety $X\left(\Sigma_{\theta}\right)$ is semi-projective, but not projective. In [HS], from $\beta^{\vee}$ and $\theta$, the authors define the hypertoric variety $Y\left(\beta^{\vee}, \theta\right)$ as the complete intersection of the toric variety $X\left(\Sigma_{\theta}\right)$ by the ideal (2.9), which is the geometric quotient $Y / G$. We have the following Proposition.

Proposition 3.1. The coarse moduli space of $\mathcal{M}(\mathcal{A})$ is $Y\left(\beta^{\vee}, \theta\right)$.

ProOF. Let $X=\left(\mathbb{C}^{2 m}-V\left(\mathcal{I}_{\theta}\right)\right)$. By the universal property of geometric quotients $([\mathrm{KM}])$, we have that $X \times_{X\left(\Sigma_{\theta}\right)} Y\left(\beta^{\vee}, \theta\right)=Y$. From Lemma 3.3 in [JT], the stabilizers of points in $X$ are the same as the stabilizers of the points in $Y$, which are determined by the box elements in the Lawrence simplicial fan and extended stacky fan. So we have the following diagram

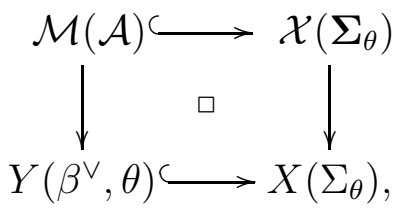

which is cartesian. The Lawrence toric variety $X\left(\Sigma_{\theta}\right)$ is the coarse moduli space of the Lawrence toric Deligne-Mumford stack $\mathcal{X}\left(\Sigma_{\theta}\right)$. So $\mathcal{M}(\mathcal{A})$ has coarse moduli space $Y\left(\beta^{\vee}, \theta\right)$.

Remark 3.2. In [HS], the authors began with the map $\beta^{\vee}$, and assumed that $D G(\beta)$ and $N$ are free. In our case $D G(\beta)$ is a finitely generated abelian group, the toric variety $X\left(\Sigma_{\theta}\right)$ is again semi-projective since $\Sigma_{\theta}$ is a semi-projective fan. The hypertoric variety $Y\left(\beta^{\vee}, \theta\right)$ is the complete intersection determined by the ideal (2.9). This reduces to the case in [HS] when $D G(\beta)$ and $N$ are free.

Independence of coorientations of hyperplanes. From (2.3), a hyperplane $H_{i}$ is naturally oriented. Changing the orientation of $H_{i}$ means changing the map $\beta$ by replacing $b_{i}$ by $-b_{i}$.

Proposition 3.3. $\mathcal{M}(\mathcal{A})$ is independent to the coorientations of the hyperplanes in the hyperplane arrangement $\mathcal{H}=\left(H_{1}, \cdots, H_{m}\right)$ corresponding to the stacky hyperplane arrangement $\mathcal{A}$.

Remark 3.4. Note that changing coorientations does change the corresponding normal fan of the weighted polytope $\boldsymbol{\Gamma}$.

ProOF. It suffices to prove the Proposition when we change the coorientation of one hyperplane, say $H_{j}$ for some $j$. Let $\mathcal{H}^{\prime}=\left(H_{1}, \cdots, H_{j}^{\prime}, \cdots, H_{m}\right)$. Then we have a new stacky hyperplane arrangement $\mathcal{A}^{\prime}=\left(N, \beta^{\prime}, \theta\right)$, where $\beta^{\prime}: \mathbb{Z}^{m} \rightarrow N$ is given by $\left\{b_{1}, \cdots,-b_{j}, \cdots, b_{m}\right\}$. Using the technique of Gale dual in [BCS], it is easy to check that if the Gale dual $\beta^{\vee}$ is given by the integral vectors $\beta^{\vee}=\left(a_{1}, \cdots, a_{m}\right)$, then the Gale dual $\left(\beta^{\prime}\right)^{\vee}$ is given by the integral vectors $\left(\beta^{\prime}\right)^{\vee}=\left(a_{1}, \cdots,-a_{j}, \cdots, a_{m}\right)$. Let $\psi: \mathbb{Z}^{m} \rightarrow \mathbb{Z}^{m}$ be the map given by $e_{i} \mapsto e_{i}$ if 
$i \neq j$ and $e_{j} \mapsto-e_{j}$, then we have the following commutative diagrams:
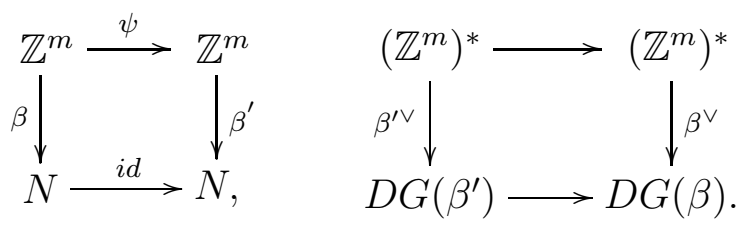

Consider the diagram

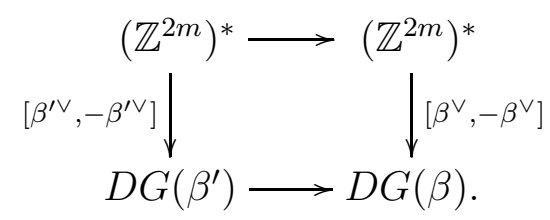

Applying $\operatorname{Hom}_{\mathbb{Z}}\left(-, \mathbb{C}^{\times}\right)$yields the following diagram of abelian groups

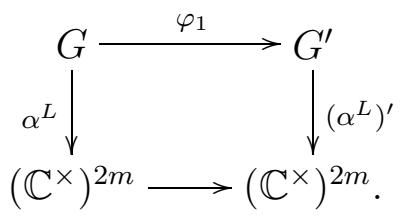

Recall that $Y$ is a subvariety of $\mathbb{C}^{2 m} \backslash V\left(\mathcal{I}_{\theta}\right)$ defined by the ideal $I_{\beta^{\vee}}$ in (2.9). When we change the coorientation of $H_{j}$, the ideals do not change, so $Y^{\prime}=Y$. By (3.1), the following diagram is Cartesian:

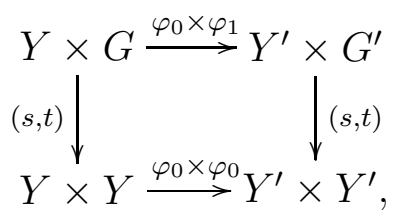

where $\varphi_{0}$ is determined by the map $\psi$. So the groupoid $Y \times G \rightrightarrows Y$ is Morita equivalent to the groupoid $Y^{\prime} \times G^{\prime} \rightrightarrows Y^{\prime}$. The stack $[Y / G]$ is isomorphic to the stack $\left[Y^{\prime} / G^{\prime}\right]$, and $\mathcal{M}(\mathcal{A}) \cong \mathcal{M}\left(\mathcal{A}^{\prime}\right)$.

Remark 3.5. Let $\boldsymbol{\Sigma}=(N, \Sigma, \beta)$ be the extended stacky fan induced by $\mathcal{A}$. The toric DM stack $\mathcal{X}(\boldsymbol{\Sigma})$ is the quotient stack $[Z / G]$, where $Z=\left(\mathbb{C}^{n} \backslash V\left(J_{\Sigma}\right)\right) \times\left(\mathbb{C}^{\times}\right)^{m-n}$ as in [Jiang], and $J_{\Sigma}$ is the square-free ideal of the fan $\Sigma$. So every hypertoric DM stack $\mathcal{M}(\mathcal{A})$ has an associated toric DM stack $\mathcal{X}(\boldsymbol{\Sigma})$ whose simplicial fan is the normal fan of the bounded polytope $\boldsymbol{\Gamma}$ in the hyperplane arrangement $\mathcal{H}$ determined by the stacky hyperplane arrangement $\mathcal{A}$. But by Proposition 3.3, $\mathcal{M}(\mathcal{A})$ does not determine $\mathcal{X}(\boldsymbol{\Sigma})$.

Example 3.6. Consider Figure 1 again. The corresponding toric variety is $\mathbb{P}^{2}$. If we change the coorientation of the hyperplane 2 , then the corresponding normal fan $\Sigma$ of $\boldsymbol{\Gamma}$ changes. The resulting toric variety is a Hirzebruch surface. So the associated toric DM stacks are different. But the hypertoric DM stacks are the same.

\section{Substacks of Hypertoric DM Stacks}

In this section we consider substacks of hypertoric DM stacks. In particular, we determine the inertia stack of a hypertoric DM stack. 
Let $\mathcal{A}=(N, \beta, \theta)$ be a stacky hyperplane arrangement and $\boldsymbol{\Sigma}=(N, \Sigma, \beta)$ the extended stacky fan induced from $\mathcal{A}$. Let $\mathcal{M}(\mathcal{A})$ denote the corresponding hypertoric DM stack. Consider the map $\beta: \mathbb{Z}^{m} \rightarrow N$ given by $\left\{b_{1}, \cdots, b_{m}\right\}$. Let $\operatorname{Cone}(\beta)$ be a partially ordered finite set of cones generated by $\bar{b}_{1}, \cdots, \bar{b}_{m}$. The partial ordering is defined by requiring that $\sigma \prec \tau$ if $\sigma$ is a face of $\tau$. We have the minimum element $\hat{0}$ which is the cone consisting of the origin. Let $\operatorname{Cone}(\bar{N})$ be the set of all convex polyhedral cones in the lattice $\bar{N}$. Then we have a map

$$
C: \text { Cone }(\beta) \longrightarrow \text { Cone }(\bar{N}),
$$

such that for any $\sigma \in \operatorname{Cone}(\beta), C(\sigma)$ is the cone in $\bar{N}$. Then $\Delta_{\beta}:=(C, \operatorname{Cone}(\beta))$ is a simplicial multi-fan in the sense of [HM].

Closed substacks. Recall that in Section 2 we have the fan $\Sigma_{\theta}$ for the Lawrence toric variety corresponding to $\pm \beta^{\vee}$. Let $\Lambda(\mathcal{B})=\left\{\bar{b}_{L, 1}, \cdots, \bar{b}_{L, m}, \bar{b}_{L, 1}^{\prime}, \cdots, \bar{b}_{L, m}^{\prime}\right\} \subset \bar{N}_{L}$ be the Lawrence lifting of $\mathcal{B}=\left\{\bar{b}_{1}, \cdots, \bar{b}_{m}\right\} \subset \bar{N}$. We have the following lemma.

Lemma 4.1. If $\sigma_{\theta}=\left(\bar{b}_{L, i_{1}}, \cdots, \bar{b}_{L, i_{k}}, \bar{b}_{L, i_{1}}^{\prime}, \cdots, \bar{b}_{L, i_{k}}^{\prime}\right)$ forms a cone in $\Sigma_{\theta}$, then $\sigma=\left(\bar{b}_{i_{1}}, \cdots, \bar{b}_{i_{k}}\right)$ forms a cone in $\Delta_{\beta}$.

Proof. This can be easily proved from the definition of fan $\Sigma_{\theta}$ in (2.6).

For a cone $\sigma$ in the multi-fan $\Delta_{\beta}$, let $\operatorname{link}(\sigma)=\left\{b_{i}: \rho_{i}+\sigma\right.$ is a cone in $\left.\Delta_{\beta}\right\}$. Then we have a quotient extended stacky fan $\Sigma / \sigma=(N(\sigma), \Sigma / \sigma, \beta(\sigma))$, where $\beta(\sigma): \mathbb{Z}^{l} \rightarrow N(\sigma)$ is given by the images of $\left\{b_{i}\right\}$ 's in $\operatorname{link}(\sigma)$. Let $s:=|\sigma|$, then $\operatorname{dim}\left(N_{\sigma}\right)=s$ since $\sigma$ is simplicial. Consider the commutative diagrams

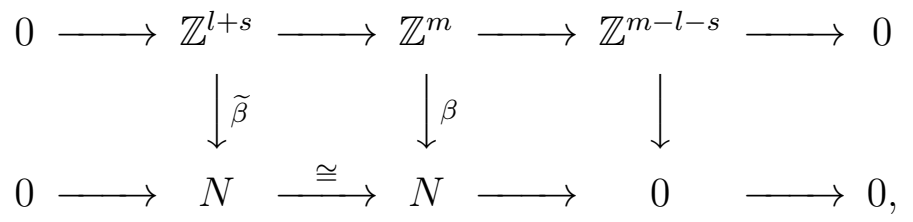

and

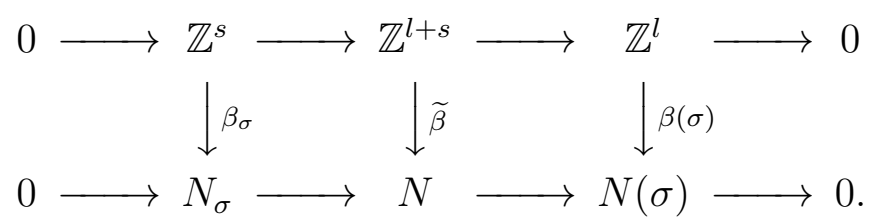

Applying the Gale dual yields

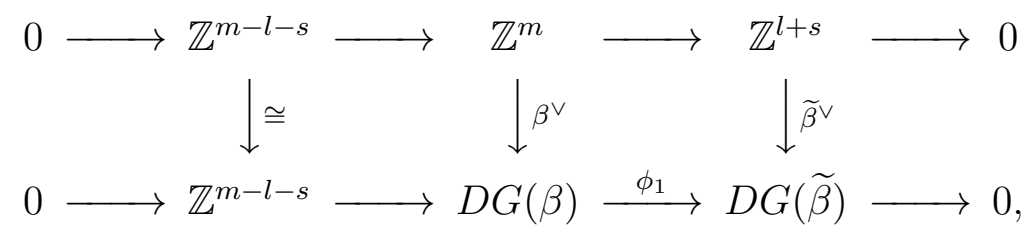

and

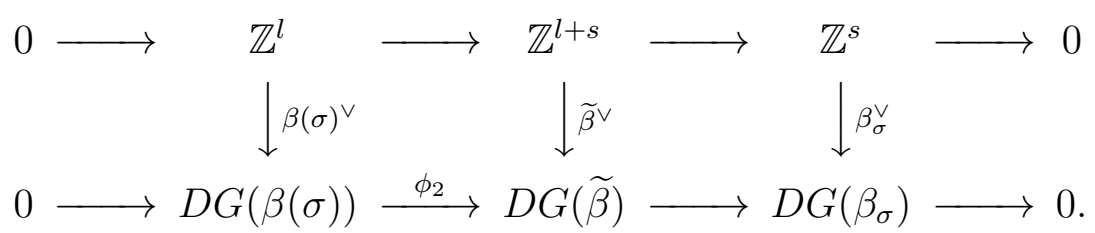


Since $\mathbb{Z}^{s} \cong N_{\sigma}$, the Gale dual $D G\left(\beta_{\sigma}\right)=0$. And again applying the $H_{o} m_{\mathbb{Z}}\left(-, \mathbb{C}^{\times}\right)$functor to the above two diagrams (4.1), (4.2) yields

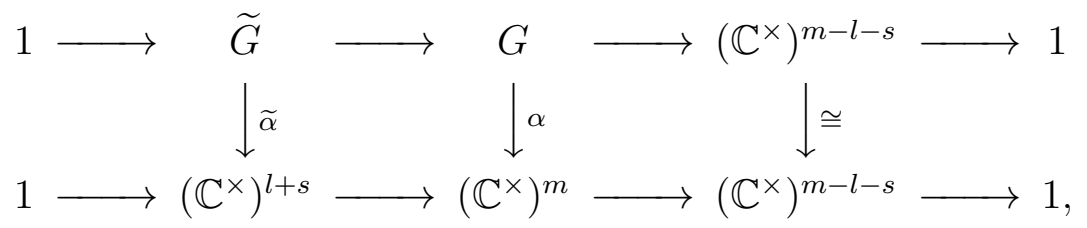

and

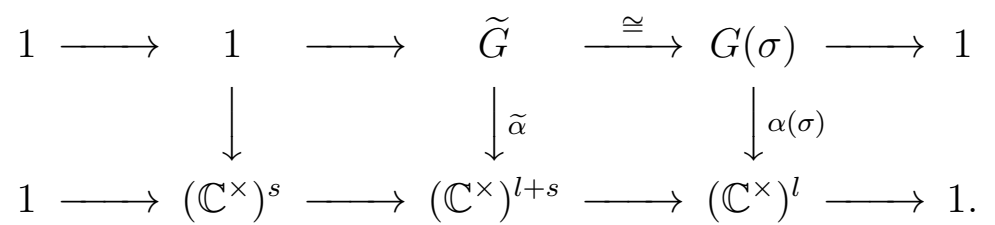

Since $\theta \in D G(\beta)$, from the map $\phi_{1}$ in (4.1) $\theta$ induces $\widetilde{\theta}$ in $D G(\widetilde{\beta})$. From the isomorphism $\phi_{2}$ in (4.2), we get $\theta(\sigma) \in D G(\beta(\sigma))$. Then $\mathcal{A}=(N, \beta, \theta)$ gives $\mathcal{A}(\sigma)=(N(\sigma), \beta(\sigma), \theta(\sigma))$ whose induced extended stacky fan is $\Sigma / \sigma$.

From (4.1), (4.2) we have the following diagrams

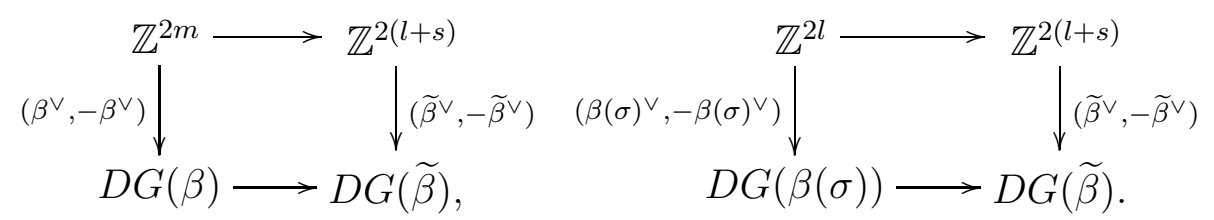

Taking $\operatorname{Hom}_{\mathbb{Z}}\left(-, \mathbb{C}^{\times}\right)$gives

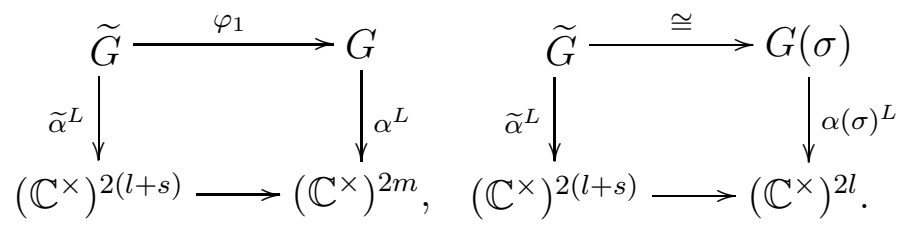

Let $X(\sigma):=\left(\mathbb{C}^{2 l} \backslash V\left(\mathcal{I}_{\theta(\sigma)}\right)\right)$ and $Y(\sigma)$ the closed subvariety of $X(\sigma)$ defined by the ideal

$$
I_{\beta(\sigma)^{\vee}}:=\left\{\sum_{i=1}^{l}\left(\beta(\sigma)^{\vee}\right)^{*}(x)_{i} z_{i} w_{i}: \forall x \in D G(\beta(\sigma))^{*}\right\},
$$

where $\left(\beta(\sigma)^{\vee}\right)^{*}: D G(\beta(\sigma))^{*} \rightarrow \mathbb{Z}^{l}$ is the dual map of $\beta(\sigma)^{\vee}$ and $\left(\beta(\sigma)^{\vee}\right)^{*}(x)_{i}$ the $i$-th component of the vector $\left(\beta(\sigma)^{\vee}\right)^{*}(x)$. Then from the definition of hypertoric DM stacks, we have $\mathcal{M}(\mathcal{A}(\sigma))=[Y(\sigma) / G(\sigma)]$. We have the following result:

Proposition 4.2. If $\sigma$ is a cone in the multi-fan $\Delta_{\beta}$, then $\mathcal{M}(\mathcal{A}(\sigma))$ is a closed substack of $\mathcal{M}(\mathcal{A})$.

Proof. Let $\mathcal{I}_{\theta}$ be the irrelevant ideal in (2.5). The hypertoric stack $\mathcal{M}(\mathcal{A})$ is the quotient stack $[Y / G]$, where $Y \subset X:=\left(\mathbb{C}^{2 m} \backslash V\left(\mathcal{I}_{\theta}\right)\right)$ is the subvariety determined by the ideal $I_{\beta^{\vee}}$ in (2.9). 
Taking duals to (4.1), (4.2) we get:

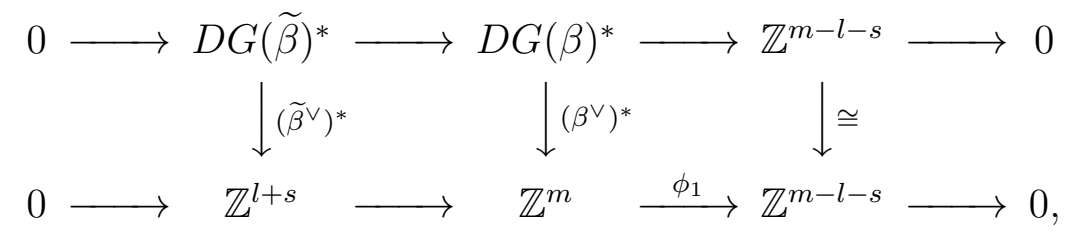

and

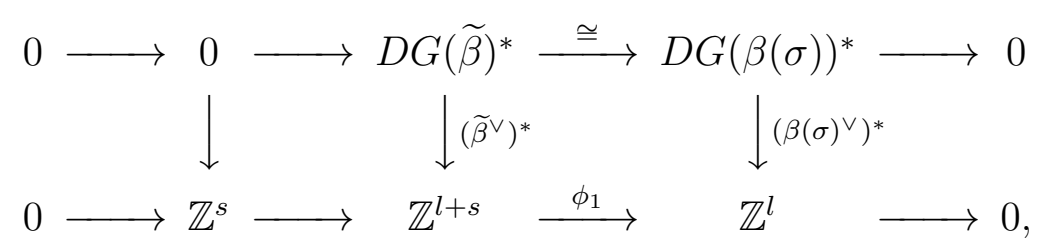

Let $W(\sigma)$ be the subvariety of $X$ defined by the ideal $J(\sigma):=\left\langle z_{i}, w_{i}: \rho_{i} \subseteq \sigma\right\rangle$. Then $W(\sigma)$ contains the $\mathbb{C}$-points $(z, w) \in \mathbb{C}^{2 m}$ such that the cone spanned by $\left\{\rho_{i}: z_{i}=w_{i}=0\right\}$ containing $\sigma$ belongs to $\Delta_{\beta}$. From Lemma 4.1, the $\mathbb{C}$-point $(z, w)$ in $W(\sigma)$ such that $\rho_{i} \nsubseteq$ $\sigma \cup \operatorname{link}(\sigma)$ implies that $z_{i} \neq 0$ or $w_{i} \neq 0$. It is clear that $W(\sigma)$ is invariant under the $G$-action defined by (2.8). Let $V(\sigma):=Y \cap W(\sigma)$. Then from (2.9), (4.7) and (4.8), (4.9), $V(\sigma) \cong Y(\sigma) \times\left(\mathbb{C}^{\times}\right)^{m-s-l} \times(0)^{m-s-l}$ and the components 0 are determined by the choice of the generic element $\theta$.

Let $\varphi_{0}: Y(\sigma) \longrightarrow V(\sigma)$ be the inclusion given by $(z, w) \longmapsto(z, w, 1,0)$. From the map $\varphi_{1}$ in (4.6), we have a morphism of groupoids $\varphi_{0} \times \varphi_{1}: Y(\sigma) \times G(\sigma) \rightrightarrows V(\sigma) \times G$ which induces a morphism of stacks $\varphi:[Y(\sigma) / G(\sigma)] \longrightarrow[V(\sigma) / G]$. To prove that it is an isomorphism, we first prove that the following diagram is cartesian:

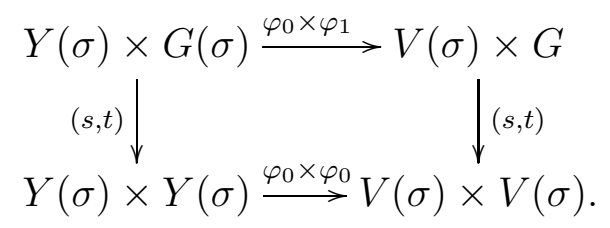

This is easy to prove. Given an element $\left(\left(z_{1}, w_{1}\right),\left(z_{2}, w_{2}\right)\right) \in Y(\sigma) \times Y(\sigma)$, under the map $\varphi_{0} \times \varphi_{0}$, we get $\left(\left(z_{1}, w_{1}, 1,0\right),\left(z_{2}, w_{2}, 1,0\right)\right) \in V(\sigma) \times V(\sigma)$. If there is an element $g \in G$ such that $g\left(z_{1}, w_{1}, 1,0\right)=\left(z_{2}, w_{2}, 1,0\right)$, then from the exact sequence in the first row of (4.3), there is an element $g(\sigma) \in G(\sigma)$ such that $g(\sigma)\left(z_{1}, w_{1}\right)=\left(z_{2}, w_{2}\right)$. Thus we have an element $\left(\left(z_{1}, w_{1}\right), g(\sigma)\right) \in Y(\sigma) \times G(\sigma)$. So the morphism $\varphi:[Y(\sigma) / G(\sigma)] \longrightarrow[V(\sigma) / G]$ is injective. Let $(z, w, s, 0)$ be an element in $V(\sigma)$, then there exists an element $g \in\left(\mathbb{C}^{\times}\right)^{m-l-s}$ such that $g(z, w, s, 0)=(z, w, 1,0)$. From (4.3), $g$ determines an element in $G$, so $\varphi$ is surjective and $\varphi$ is an isomorphism. Clearly the stack $[V(\sigma) / G]$ is a closed substack of $\mathcal{M}(\mathcal{A})$, so the stack $\mathcal{M}(\mathcal{A}(\sigma))=[Y(\sigma) / G(\sigma)]$ is also a closed substack of $\mathcal{M}(\mathcal{A})$.

Open substacks. We now study open substacks of $\mathcal{M}(\mathcal{A})$. Let $\sigma$ be a top dimensional cone in $\Delta_{\beta}$. Then $\sigma=\left(\mathbb{Z}^{d}, \sigma, \beta_{\sigma}\right)$ is a stacky fan, where $\beta_{\sigma}: \mathbb{Z}^{d} \rightarrow N$ is given by $b_{i}$ for $\rho_{i} \subseteq \sigma$. Since $N$ has rank $d$, we find that $D G\left(\beta_{\sigma}\right)$ is a finite abelian group. So in this case the generic element $\theta$ induces zero in $D G\left(\beta_{\sigma}\right)$. This is the degenerate case, which means that the corresponding ideal $(\underline{2.9})$ is zero. Thus

$$
Y_{\sigma}=\mathbb{C}^{2 d}
$$


Note that $G_{\sigma}=H_{0} m_{\mathbb{Z}}\left(D G\left(\beta_{\sigma}\right), \mathbb{C}^{\times}\right)$is a finite abelian group. According to the construction of hypertoric DM stack in Section 2, the hypertoric DM stack $\mathcal{M}(\sigma)$ associated to $\sigma$ is the quotient stack $\left[Y_{\sigma} / G_{\sigma}\right]$ which can be regarded as a local chart of the hypertoric orbifold $[Y / G]$.

Proposition 4.3. If $\sigma$ is a top-dimensional cone in the multi-fan $\Delta_{\beta}$, then $\mathcal{M}(\sigma)$ is an open substack of $\mathcal{M}(\mathcal{A})$.

Proof. Since $\sigma$ is a top dimensional cone in $\Delta_{\beta}$, from $(2.2)$ we get a basis $C$ of $\overline{D G(\beta)}$. Let $U_{\sigma}$ be the open subvariety of $\mathbb{C}^{2 m} \backslash V\left(\mathcal{I}_{\theta}\right)$ defined by the monomials $\prod C(\theta)$ in (2.5). Let $V_{\sigma}=U_{\sigma} \cap Y$, i.e. the points in $U_{\sigma}$ staisfying (2.9). Then we have the groupoid $V_{\sigma} \times G \rightrightarrows V_{\sigma}$ associated to the action of $G$ on $V_{\sigma}$. It is clear that this groupoid defines an open substack of $\mathcal{M}(\mathcal{A})$. Next we show that this substack is isomorphic to $\mathcal{M}(\sigma)$.

Consider the following commutative diagram:

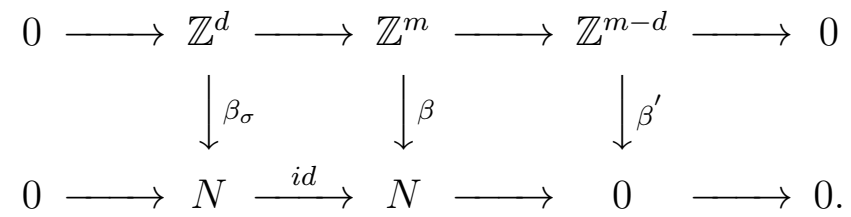

Applying Gale dual and $\operatorname{Hom}_{\mathbb{Z}}\left(-, \mathbb{C}^{\times}\right)$, we obtain

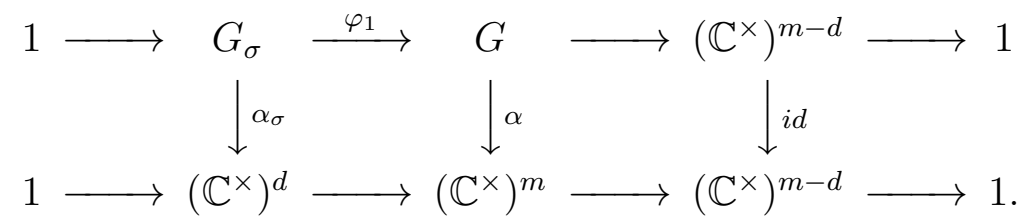

We construct a morphism $\varphi_{0}: Y_{\sigma} \rightarrow V_{\sigma}$. For $\rho_{j} \nsubseteq \sigma$, we set $z_{j}=1$ if $z_{j}$ is component of a monomial of $C(\theta)$ in (2.5) or $w_{j}=1$ if $w_{j}$ is component of a monomial of $C(\theta)$ in (2.5), then from (2.9), the corresponding $w_{j}$ or $z_{j}$ can be represented as linear component of $\left\{z_{i} w_{i}\right\}$ for $\rho_{i} \subseteq \sigma$. Let $\widetilde{\varphi}_{0}: \mathbb{C}^{2 d} \rightarrow U_{\sigma}$ be the morphism given by $z_{i}, w_{i} \longmapsto z_{i}, w_{i}$ for $\rho_{i} \subseteq \sigma$, and $z_{j}, w_{j}$ to the corresponding 1 or linear combination of $\left\{z_{i} w_{i}\right\}$ for $\rho_{i} \subseteq \sigma$ in the above analysis. Then $\widetilde{\varphi}_{0}$ induces a morphism $\varphi_{0}: Y_{\sigma} \rightarrow V_{\sigma}$. Hence we have a morphism of groupoids

$$
\Phi:=\left(\varphi_{0} \times \varphi_{0}, \varphi_{0} \times \varphi_{1}\right):\left[Y_{\sigma} \times G_{\sigma} \rightrightarrows Y_{\sigma}\right] \longrightarrow\left[V_{\sigma} \times G \rightrightarrows V_{\sigma}\right],
$$

where $\varphi_{1}$ is the morphism in (4.10). This morphism determines a morphism of the associated stacks. The isomorphism of these two stacks comes from the following Cartesian diagram:

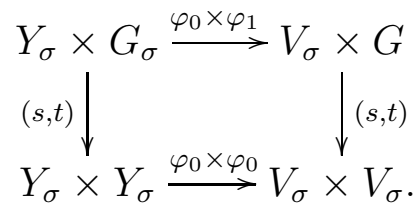

Inertia stacks. Let $N_{\sigma}$ be the sublattice generated by $\sigma$, and $N(\sigma):=N / N_{\sigma}$. Note that when $\sigma$ is a top dimensional cone, $N(\sigma)$ is the local orbifold group in the local chart of the coarse moduli space of the hypertoric toric DM stack. Namely:

Lemma 4.4. Let $\sigma$ be a top-dimensional cone in the multi-fan $\Delta_{\beta}$. Then $G_{\sigma} \cong N(\sigma)$. 
Proof. The proof is the same as the proof for a top dimensional cone in a simplicial fan in Proposition 4.3 in [BCS].

Recall that $G$ acts on $\left(\mathbb{C}^{\times}\right)^{2 m}$ via the map $\alpha^{L}: G \rightarrow\left(\mathbb{C}^{\times}\right)^{2 m}$ in (2.8). We write

$$
\alpha^{L}(g)=\left(\alpha_{1}^{L}(g), \cdots, \alpha_{m}^{L}(g), \alpha_{1+m}^{L}(g), \cdots, \alpha_{2 m}^{L}(g)\right)
$$

Lemma 4.5. Let $(z, w) \in Y$ be a point fixed by $g \in G$. If $\alpha_{i}^{L}(g) \neq 1$, then $z_{i}=w_{i}=0$.

Proof. Since $G$ acts on $\mathbb{C}^{2 m}$ through the matrix $\beta_{L}^{\vee}=\left[\beta^{\vee},-\beta^{\vee}\right]$ in (2.7), we have that $\alpha_{i+m}^{L}(g)=\alpha_{i}^{L}(g)^{-1}$. The Lemma follows immediately.

Given the multi-fan $\Delta_{\beta}$, we consider the pairs $(v, \sigma)$, where $\sigma$ is a cone in $\Delta_{\beta}, v \in N$ such that $\bar{v}=\sum_{\rho_{i} \subseteq \sigma} \alpha_{i} b_{i}$ for $0<\alpha_{i}<1$. Note that $\sigma$ is the minimal cone in $\Delta_{\beta}$ satisfying the above condition. Let $\operatorname{Box}\left(\Delta_{\beta}\right)$ be the set of all such pairs $(v, \sigma)$.

Proposition 4.6. There is an one-to-one correspondence between $g \in G$ with nonempty fixed point set and $(v, \sigma) \in \operatorname{Box}\left(\Delta_{\beta}\right)$. Moreover, for such g and $(v, \sigma)$ we have $\left[Y^{g} / G\right] \cong \mathcal{M}(\mathcal{A}(\sigma))$.

Proof. Let $(v, \sigma) \in \operatorname{Box}\left(\Delta_{\beta}\right)$. Since $\sigma$ is contained in a top dimensional cone $\tau$ in $\Delta_{\beta}$, we have $v \in N(\tau)$. By Lemma 4.4, $N(\tau) \cong G_{\tau}$. Hence $v$ determines an element in $G_{\tau}$. Using the morphism $\varphi_{1}$ in (4.10), we see that $g$ fixes a point in $Y$.

Conversely, suppose $g \in G$ fixes a point $(z, w)$ in $Y$, where $(z, w) \in \mathbb{C}^{2 m}$. By Lemma 4.5, the point $(z, w)$ satisfies the condition that if $\alpha_{i}^{L}(g) \neq 1$ then $z_{i}=w_{i}=0$. From the definition of $\mathbb{C}^{2 m} \backslash V\left(\mathcal{I}_{\theta}\right)$, there is a cone in $\Sigma_{\theta}$ containing the rays for which $z_{i}=w_{i}=0$. By Lemma 4.1, the rays $\rho_{i}$ for which $z_{i}=0$ is a cone in $\Delta_{\beta}$ which we call $\sigma$. So $g$ stabilizes $Y_{\tau}=\mathbb{C}^{2 d}$ in $V_{\tau}$ through $\varphi_{0}$ in (4.11) for any top dimensional cone $\tau$ containing $\sigma$, and $g$ corresponds to an element $(v, \sigma) \in \operatorname{Box}\left(\Delta_{\beta}\right)$. From the definition of $W(\sigma)$ and $V(\sigma)$ in Proposition 4.2, we have $W(\sigma) \cong Y^{g}$ and $[V(\sigma) / G] \cong\left[Y^{g} / G\right]$ which is $\mathcal{M}(\mathcal{A}(\sigma))$.

We determine the inertia stack of a hypertoric DM stack.

Proposition 4.7. The inertia stack of $\mathcal{M}(\mathcal{A})$ is given by

$$
I(\mathcal{M}(\mathcal{A}))=\coprod_{(v, \sigma) \in \operatorname{Box}\left(\Delta_{\beta}\right)} \mathcal{M}(\mathcal{A}(\sigma)) .
$$

Proof. The hypertoric DM stack $\mathcal{M}(\mathcal{A})=[Y / G]$ is a quotient stack. Its inertia stack is determined as

$$
I(\mathcal{M}(\mathcal{A}))=\left[\left(\coprod_{g \in G} Y^{g}\right) / G\right] .
$$

By Proposition 4.6, the stack $\left[Y^{g} / G\right]$ is isomorphic to the stack $\mathcal{M}(\mathcal{A}(\sigma))$ for some $(v, \sigma) \in$ $\operatorname{Box}\left(\Delta_{\beta}\right)$.

Example 4.8. Let $\boldsymbol{\Sigma}=(N, \Sigma, \beta)$ be an extended stacky fan, where $N=\mathbb{Z}^{2}$, the simplicial 


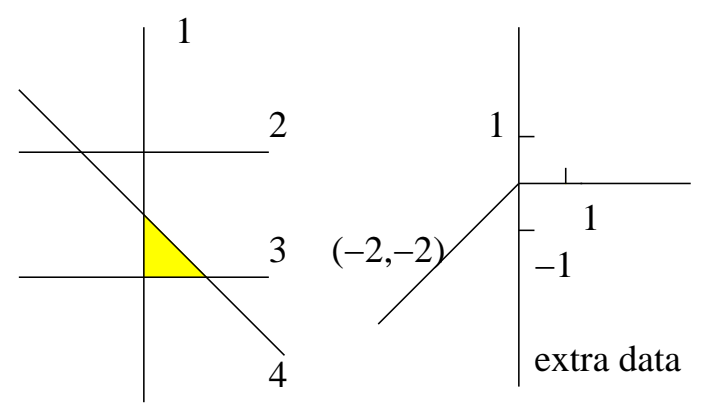

Figure 2: The correspondence of the hyperplane arrangement and an extended stacky fan

fan $\Sigma$ is the fan of weighted projective plane $\mathbb{P}(1,2,2)$, and $\beta: \mathbb{Z}^{4} \rightarrow N$ is given by the vectors $\left\{b_{1}=(1,0), b_{2}=(0,1), b_{3}=(-2,-2), b_{4}=(0,-1)\right\}$, where $b_{1}, b_{2}, b_{3}$ are the generators of the rays in $\Sigma$. Choose generic element $\theta=(1,1) \in D G(\beta) \cong \mathbb{Z}^{2}$. Then $\mathcal{A}=(N, \beta, \theta)$ is the stacky hyperplane arrangement whose induced extended stacky fan is $\Sigma$. A lifting of $\theta$ in $\mathbb{Z}^{4}$ through the Gale dual map $\beta^{\vee}$ is $r=(1,1,-3,0)$. The corresponding hyperplane arrangement $\mathcal{H}=\left(H_{1}, H_{2}, H_{3}, H_{4}\right)$ consists of 4 lines, see Figure 2. Take $v=\frac{1}{2} b_{3}$, then $(v, \sigma) \in \operatorname{Box}\left(\Delta_{\beta}\right)$, where $\sigma$ is the ray generated by $b_{3}$. Consider the following diagram

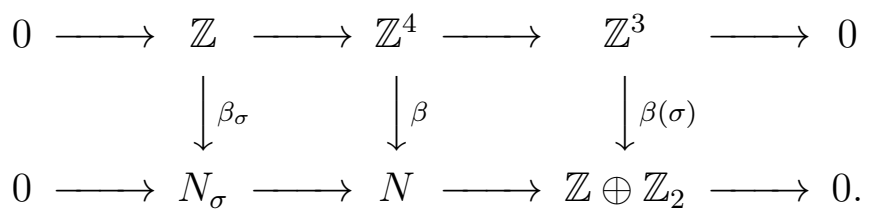

We have the quotient extended stacky fan $\Sigma / \sigma=(N(\sigma), \Sigma / \sigma, \beta(\sigma))$, where $\beta(\sigma): \mathbb{Z}^{3} \rightarrow N(\sigma)$ is given by the vectors $\{(1,0),(-1,0),(1,0)\}$, and $(1,0)$ is the extra data in the quotient extended stacky fan. Taking Gale dual, we get

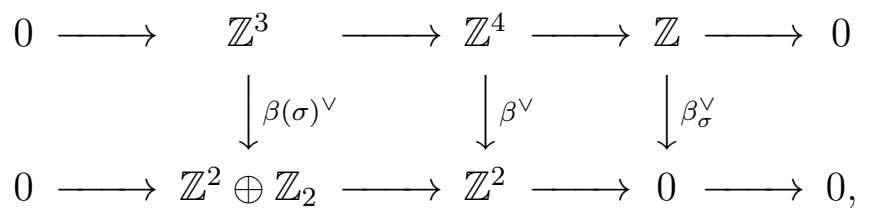

where $\beta^{\vee}$ is given by the matrix $\left[\begin{array}{llll}2 & 2 & 1 & 0 \\ 0 & 1 & 0 & 1\end{array}\right]$ and $\beta(\sigma)^{\vee}$ is given by $\left[\begin{array}{lll}2 & 2 & 1 \\ 0 & 1 & 0\end{array}\right]$. The associated generic element $\theta(\sigma)=(1,1,0)$ and the lifting of $\theta(\sigma)$ in $\mathbb{Z}^{3}$ is $r(\sigma)=(1,1,-3)$. So the quotient hyperplane arrangement $\mathcal{A}(\sigma)=(N(\sigma), \beta(\sigma), \theta(\sigma))$ is a line with three distinct points $\{-1,1,3\}$. The bounded polyhedron of this hyperplane arrangement is two segments intersecting at one point, see Figure 3.

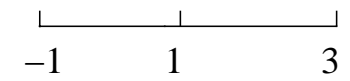

Figure 3: The bounded polyhedron

The core of $\mathcal{M}(\mathcal{A}(\sigma))$ corresponds to these two segments, hence is two $\mathbb{P}^{1}$ 's meeting at one point. Adding the stacky structure the twisted sector $\mathcal{M}(\mathcal{A}(\sigma))$ corresponding to the element $v$ is the trivial $\mu_{2}$-gerbe over the crepant resolution of the stack $\left[\mathbb{C}^{2} / \mathbb{Z}_{3}\right]$. 


\section{Orbifold Chow Ring of $\mathcal{M}(\mathcal{A})$}

In this section we discuss the orbifold Chow ring of hypertoric DM stacks. We determine its module structure, then compute the orbifold cup product.

5.1. The module structure. We first consider the ordinary Chow ring for hypertoric DM stacks. According to $[\mathrm{K}]$, the cohomology ring of $\mathcal{M}(\mathcal{A})$ is generated by the Chern classes of some line bundles defined as follows. Applying $\operatorname{Hom}_{\mathbb{Z}}\left(-, \mathbb{C}^{\times}\right)$to $(2.2)$, we have

$$
1 \longrightarrow \mu \longrightarrow G \stackrel{\alpha}{\longrightarrow}\left(\mathbb{C}^{\times}\right)^{m} \longrightarrow T \longrightarrow 1 \text {. }
$$

Definition 5.1. For every $b_{i}$ in the stacky hyperplane arrangement, define the line bundle $L_{i}$ over $\mathcal{M}(\mathcal{A})$ to be the trivial line bundle $Y \times \mathbb{C}$ with the $G$-action on $\mathbb{C}$ defined via the $i$-th component of the morphism $\alpha: G \rightarrow\left(\mathbb{C}^{\times}\right)^{m}$ in the above exact sequence.

For any $c \in N$, there is a cone $\sigma \in \Delta_{\beta}$ such that $\bar{c}=\sum_{\rho_{i} \subseteq \sigma} \alpha_{i} \bar{b}_{i}$ where $\alpha_{i}>0$ are rational numbers. Let $N^{\Delta_{\beta}}$ denote all the pairs $(c, \sigma)$. Then $N^{\Delta_{\beta}}$ gives rise a group ring

$$
\mathbb{Q}\left[\Delta_{\beta}\right]=\bigoplus_{(c, \sigma) \in N^{\Delta_{\beta}}} \mathbb{Q} \cdot y^{(c, \sigma)},
$$

where $y$ is a formal variable. By abuse of notation, we write $y^{\left(b_{i}, \rho_{i}\right)}$ as $y^{b_{i}}$. The multiplication is given in terms of the ceiling function for fans which we define below. Since the multi-fan $\Delta_{\beta}$ is simplicial, we have the following Lemma.

Lemma 5.2. For any $c \in N$, there exists a unique cone $\sigma \in \Delta_{\beta}$ and $(v, \tau) \in \operatorname{Box}\left(\Delta_{\beta}\right)$ such that $\tau \subseteq \sigma$ and

$$
c=v+\sum_{\rho_{i} \subseteq \sigma} m_{i} b_{i}
$$

where $m_{i} \in \mathbb{Z}_{\geq 0}$.

Definition 5.3. $(v, \tau)$ is called the fractional part of $(c, \sigma)$.

Now for $(c, \sigma) \in N^{\Delta_{\beta}}$, from Lemma [5.2, we write $c=v+\sum_{\rho_{i} \subseteq \sigma} m_{i} b_{i}$, where $m_{i}$ 's are nonnegative integers. We define the ceiling function $\lceil c\rceil_{\sigma}$ by

$$
\lceil c\rceil_{\sigma}=\sum_{\rho_{i} \subseteq \tau} b_{i}+\sum_{\rho_{i} \subseteq \sigma} m_{i} b_{i} .
$$

Note that if $\bar{v}=0,\lceil c\rceil_{\sigma}=\sum_{\rho_{i} \subseteq \sigma} m_{i} b_{i}$. For two pairs $\left(c_{1}, \sigma_{1}\right),\left(c_{2}, \sigma_{2}\right)$, if $\sigma_{1} \cup \sigma_{2}$ is a cone in $\Delta_{\beta}$, define $\epsilon\left(c_{1}, c_{2}\right):=\left\lceil c_{1}\right\rceil_{\sigma_{1}}+\left\lceil c_{2}\right\rceil_{\sigma_{2}}-\left\lceil c_{1}+c_{2}\right\rceil_{\sigma_{1} \cup \sigma_{2}}$. Let $\sigma_{\epsilon} \subseteq \sigma_{1} \cup \sigma_{2}$ be the minimal cone in $\Delta_{\beta}$ containing $\epsilon\left(c_{1}, c_{2}\right)$ so that $\left(\epsilon\left(c_{1}, c_{2}\right), \sigma_{\epsilon}\right) \in N^{\Delta_{\beta}}$. The ceiling function $\lceil c\rceil_{\sigma}$ is an integral linear combination of $b_{i}$ 's for $\rho_{i} \subseteq \sigma$. We define the grading on $\mathbb{Q}\left[\Delta_{\beta}\right]$ as follows. For any $(c, \sigma)$, write $c=v+\sum_{\rho_{i} \subseteq \sigma} m_{i} b_{i}$, then

$$
\operatorname{deg}\left(y^{(c, \sigma)}\right):=|\tau|+\sum_{\rho_{i} \subseteq \sigma} m_{i},
$$

where $|\tau|$ is the dimension of $\tau$. Let $\operatorname{Cir}\left(\Delta_{\beta}\right)$ be the ideal in $\mathbb{Q}\left[\Delta_{\beta}\right]$ generated by the elements in (1.2). The multiplication $y^{\left(c_{1}, \sigma_{1}\right)} \cdot y^{\left(c_{2}, \sigma_{2}\right)}$ is defined by (1.1).

Lemma 5.4. The multiplication (1.1) is associative. 
Proof. For any three pairs $\left(c_{1}, \sigma_{1}\right),\left(c_{2}, \sigma_{2}\right),\left(c_{3}, \sigma_{3}\right)$, if $\sigma_{1} \cup \sigma_{2} \cup \sigma_{3}$ is a cone in $\Delta_{\beta}$, let $\sigma \subseteq \sigma_{1} \cup \sigma_{2} \cup \sigma_{3}$ be the minimal cone in $\Delta_{\beta}$ containing

$$
\epsilon\left(c_{1}, c_{2}, c_{3}\right):=\left\lceil c_{1}\right\rceil_{\sigma_{1}}+\left\lceil c_{2}\right\rceil_{\sigma_{2}}+\left\lceil c_{3}\right\rceil_{\sigma_{3}}-\left\lceil c_{1}+c_{2}+c_{3}\right\rceil_{\sigma_{1} \cup \sigma_{2} \cup \sigma_{3}},
$$

such that $\left(\epsilon\left(c_{1}, c_{2}, c_{3}\right), \sigma\right) \in N^{\Delta_{\beta}}$. Then we check from the properties of ceiling function that $\left(y^{\left(c_{1}, \sigma_{1}\right)} \cdot y^{\left(c_{2}, \sigma_{2}\right)}\right) \cdot y^{\left(c_{3}, \sigma_{3}\right)}$ and $y^{\left(c_{1}, \sigma_{1}\right)} \cdot\left(y^{\left(c_{2}, \sigma_{2}\right)} \cdot y^{\left(c_{3}, \sigma_{3}\right)}\right)$ are both equal to

$$
\begin{cases}(-1)^{|\sigma|} y^{\left(c_{1}+c_{2}+c_{3}+\epsilon\left(c_{1}, c_{2}, c_{3}\right), \sigma_{1} \cup \sigma_{2} \cup \sigma_{3}\right)} & \text { if } \sigma_{1} \cup \sigma_{2} \cup \sigma_{3} \text { is a cone in } \Delta_{\beta}, \\ 0 & \text { otherwise }\end{cases}
$$

It is easy to check that the product preserves the grading, and the proof is left to the readers.

Consider the map $\beta: \mathbb{Z}^{m} \rightarrow N$ which is given by $\left\{b_{1}, \cdots, b_{m}\right\}$. We take $\{1, \cdots, m\}$ as the vertex set. The matroid complex $M_{\beta}$ is defined using $\beta$ by requiring that $F \in M_{\beta}$ iff the normal vectors $\left\{\bar{b}_{i}\right\}_{i \in F}$ are linearly independent in $\bar{N}$. The Stanley-Reisner ring of the matroid $M_{\beta}$ is

$$
\mathbb{Q}\left[M_{\beta}\right]=\frac{\mathbb{Q}\left[y^{b_{1}}, \cdots, y^{b_{m}}\right]}{I_{M_{\beta}}},
$$

where $I_{M_{\beta}}$ is the matroid ideal generated by the set of square-free monomials

$$
\left\{y^{b_{i_{1}}} \cdots y^{b_{i_{k}}} \mid \bar{b}_{i_{1}}, \cdots, \bar{b}_{i_{k}} \text { linearly dependent in } \bar{N}\right\} \text {. }
$$

It is clear that $\mathbb{Q}\left[M_{\beta}\right]$ is a subring of $\mathbb{Q}\left[\Delta_{\beta}\right]$ under the injection $y^{b_{i}} \longmapsto y^{\left(b_{i}, \rho_{i}\right)}$.

Lemma 5.5. Let $\mathcal{A}=(N, \beta, \theta)$ be a stacky hyperplane arrangement and $\mathcal{M}(\mathcal{A})$ the corresponding hypertoric DM stack, then we have an isomorphism of graded rings

$$
A^{*}(\mathcal{M}(\mathcal{A})) \cong \frac{\mathbb{Q}\left[M_{\beta}\right]}{\operatorname{Cir}\left(\Delta_{\beta}\right)}
$$

given by $c_{1}\left(L_{i}\right) \mapsto y^{b_{i}}$, where $\operatorname{Cir}\left(\Delta_{\beta}\right)$ is the ideal generated by elements in (1.2).

Proof. Let $Y\left(\beta^{\vee}, \theta\right)$ be the coarse moduli space of the hypertoric DM stack $\mathcal{M}(\mathcal{A})$. By [HS], we have

$$
A^{*}\left(Y\left(\beta^{\vee}, \theta\right)\right) \cong \frac{\mathbb{Q}\left[M_{\beta}\right]}{\operatorname{Cir}\left(\Delta_{\beta}\right)},
$$

given by $D_{i} \mapsto y^{b_{i}}$, where $D_{i}$ is the $T$-equivariant Weil divisor on $Y\left(\beta^{\vee}, \theta\right)$. Let $a_{i}$ be the first lattice vector in the ray generated by $b_{i}$, then $\bar{b}_{i}=l_{i} a_{i}$ for some positive integer $l_{i}$. By [V], the Chow ring of the stack $\mathcal{M}(\mathcal{A})$ is isomorphic to the Chow ring of its coarse moduli space $Y(A, \theta)$ via $c_{1}\left(L_{i}\right) \mapsto l_{i}^{-1} \cdot D_{i}$, and $\sum_{i=1}^{m} e\left(a_{i}\right) l_{i} y^{b_{i}}=\sum_{i=1}^{m} e\left(b_{i}\right) y^{b_{i}}$ for $e \in N^{*}$.

Remark 5.6. Note that we have an isomorphism:

$$
\frac{\mathbb{Q}\left[M_{\beta}\right]}{\operatorname{Cir}\left(\Delta_{\beta}\right)} \cong \frac{\mathbb{Q}\left[y^{b_{1}}, \cdots, y^{b_{m}}, y^{b_{1}^{\prime}}, \cdots, y^{b_{m}^{\prime}}\right]}{\left(\operatorname{Cir}\left(\Delta_{\beta}\right), y^{b_{1}}+y^{b_{1}^{\prime}}, \cdots, y^{b_{m}}+y^{b_{m}^{\prime}}\right)}
$$

for which the right side is the Chow ring of the Lawrence toric DM stack $\mathcal{X}\left(\Sigma_{\theta}\right)$ defined in Definition [2.6, see [HS]. So in the DM stack situation $A^{*}(\mathcal{M}(\mathcal{A})) \cong A^{*}\left(\mathcal{X}\left(\boldsymbol{\Sigma}_{\theta}\right)\right)$. This point of view will be covered and generalized in [JT]. 
Let $A_{\text {orb }}^{*}(\mathcal{M}(\mathcal{A}))$ denote the orbifold Chow ring of $\mathcal{M}(\mathcal{A})$, which by definition is $A^{*}(I(\mathcal{M}(\mathcal{A})))$ as a group. By Proposition 4.7, we have

$$
A^{*}(I(\mathcal{M}(\mathcal{A}))) \cong \bigoplus_{(v, \sigma) \in \operatorname{Box}\left(\Delta_{\beta}\right)} A^{*}(\mathcal{M}(\mathcal{A}(\sigma)))
$$

For $(v, \sigma) \in \operatorname{Box}\left(\Delta_{\beta}\right)$, there is an exact sequence of vector bundles,

$$
\left.0 \rightarrow T \mathcal{M}(\mathcal{A}(\sigma)) \rightarrow T \mathcal{M}(\mathcal{A})\right|_{\mathcal{M}(\mathcal{A}(\sigma))} \rightarrow N_{v} \rightarrow 0
$$

where $N_{v}$ denotes the normal bundle of $\mathcal{M}(\mathcal{A}(\sigma))$ in $\mathcal{M}(\mathcal{A})$. On the other hand, there is a surjective morphism

$$
\bigoplus_{i=1}^{m}\left(L_{i} \oplus L_{i}^{-1}\right) \rightarrow T \mathcal{M}(\mathcal{A})
$$

Restricting this to $\mathcal{M}(\mathcal{A}(\sigma))$ yields a surjective map

$$
\bigoplus_{\rho_{i} \subset \sigma(\bar{v})}\left(L_{i} \oplus L_{i}^{-1}\right) \rightarrow N_{v}
$$

Moreover, the element in the local group represented by $v$ acts trivially on the kernel. Let $v$ act on $L_{i}$ with eigenvalue $e^{2 \pi \sqrt{-1} w_{i}}$, where $w_{i} \in[0,1) \cap \mathbb{Q}$. It follows that the age function on the component $\mathcal{M}(\mathcal{A}(\sigma))$ assumes the value

$$
\sum_{\rho_{i} \subset \sigma}\left(w_{i}+\left(1-w_{i}\right)\right)=|\sigma|
$$

Hence $A_{\text {orb }}^{*}(\mathcal{M}(\mathcal{A}))$ as a graded module coincides with

$$
\bigoplus_{(v, \sigma) \in \operatorname{Box}\left(\Delta_{\beta}\right)} A^{*}(\mathcal{M}(\mathcal{A}(\sigma)))[|\sigma|] .
$$

Note that $A_{\text {orb }}^{*}(\mathcal{M}(\mathcal{A}))$ is $\mathbb{Z}$-graded, due to the fact that $\mathcal{M}(\mathcal{A})$ is hyperkähler.

Again since the multi-fan $\Delta_{\beta}$ is simplicial, we have the following result, similar to Lemma 4.6 in Jiang.

Lemma 5.7. Let $\tau$ be a cone in the multi-fan $\Delta_{\beta}$. If $\left\{\rho_{1}, \cdots, \rho_{t}\right\} \subset \operatorname{link}(\tau)$, and suppose $\rho_{1}, \cdots, \rho_{t}$ are contained in a cone $\sigma \in \Delta_{\beta}$. Then $\sigma \cup \tau$ is contained in a cone of $\Delta_{\beta}$.

Proposition 5.8. Let $\mathcal{M}(\mathcal{A})$ be the hypertoric DM stack associated to the stacky hyperplane arrangement $\mathcal{A}$, then we have an isomorphism of graded $A^{*}(\mathcal{M}(\mathcal{A}))$-modules:

$$
\frac{\mathbb{Q}\left[\Delta_{\beta}\right]}{\operatorname{Cir}\left(\Delta_{\beta}\right)} \cong \bigoplus_{(v, \sigma) \in \operatorname{Box}\left(\Delta_{\beta}\right)} A^{*}(\mathcal{M}(\mathcal{A}(\sigma)))\left[\operatorname{deg}\left(y^{(v, \sigma)}\right)\right]
$$

Proof. We use arguments similar to those in Proposition 4.7 of [Jiang. From Lemma 5.2 it is easy to see that

$$
\mathbb{Q}\left[\Delta_{\beta}\right] \cong \bigoplus_{(v, \sigma) \in \operatorname{Box}\left(\Delta_{\beta}\right)} y^{(v, \sigma)} \cdot \mathbb{Q}\left[M_{\beta}\right]
$$

Consider $\bigoplus_{(v, \sigma) \in \operatorname{Box}\left(\Delta_{\beta}\right)} y^{(v, \sigma)} \cdot \operatorname{Cir}\left(\Delta_{\beta}\right)$. It is an ideal of the ring $\bigoplus_{(v, \sigma) \in \operatorname{Box}\left(\Delta_{\beta}\right)} y^{(v, \sigma)} \cdot \mathbb{Q}\left[M_{\beta}\right]$, so

$$
\frac{\mathbb{Q}\left[\Delta_{\beta}\right]}{\operatorname{Cir}\left(\Delta_{\beta}\right)} \cong \bigoplus_{(v, \sigma) \in \operatorname{Box}\left(\Delta_{\beta}\right)} \frac{y^{(v, \sigma)} \cdot \mathbb{Q}\left[M_{\beta}\right]}{y^{(v, \sigma)} \cdot \operatorname{Cir}\left(\Delta_{\beta}\right)}
$$


For an element $(v, \sigma) \in \operatorname{Box}\left(\Delta_{\beta}\right)$, let $\rho_{1}, \cdots, \rho_{l} \in \operatorname{link}(\sigma)$. Then we have an induced matroid complex $M_{\beta(\sigma)}$, where $\beta(\sigma)$ is the map in the quotient stacky hyperplane arrangement $\mathcal{A}(\sigma)$ and the quotient extended stacky fan $\Sigma / \sigma$. Similarly from $\beta(\sigma)$, we have multi-fan $\Delta_{\beta(\sigma)}$ in $\overline{N(\sigma)}$. By Lemma 5.5, $A^{*}(\mathcal{M}(\mathcal{A}(\sigma))) \cong \mathbb{Q}\left[M_{\beta(\sigma)}\right] / \operatorname{Cir}\left(\Delta_{\beta(\sigma)}\right)$. For any element $(v, \sigma) \in \operatorname{Box}\left(\Delta_{\beta}\right)$, we construct an isomorphism

$$
\Psi_{v}: \frac{\mathbb{Q}\left[M_{\beta(\sigma)}\right]}{\operatorname{Cir}\left(\Delta_{\beta(\sigma)}\right)}\left[\operatorname{deg}\left(y^{(v, \sigma)}\right)\right] \longrightarrow \frac{y^{(v, \sigma)} \cdot \mathbb{Q}\left[M_{\beta}\right]}{y^{(v, \sigma)} \cdot \operatorname{Cir}\left(\Delta_{\beta}\right)} .
$$

as follows. Consider the quotient stacky hyperplane arrangement $\mathcal{A}(\sigma)=(N(\sigma), \beta(\sigma), \theta(\sigma))$. The hypertoric DM stack $\mathcal{M}(\mathcal{A}(\sigma))$ is a closed substack of $\mathcal{M}(\mathcal{A})$. Consider the morphism $i: \mathbb{Q}\left[y^{\widetilde{b}_{1}}, \ldots, y^{\widetilde{b}_{l}}\right] \rightarrow \mathbb{Q}\left[y^{b_{1}}, \ldots, y^{b_{m}}\right]$ given by $y^{\widetilde{b}_{i}} \mapsto y^{b_{i}}$. By Lemma 5.7, it is easy to check that the ideal $I_{M_{\beta(\sigma)}}$ is mapped to the ideal $I_{M_{\beta}}$, so we have a morphism $\mathbb{Q}\left[M_{\beta(\sigma)}\right] \rightarrow \mathbb{Q}\left[M_{\beta}\right]$. Since $\mathbb{Q}\left[M_{\beta}\right]$ is a subring of $\mathbb{Q}\left[\Delta_{\beta}\right]$. Let $\widetilde{\Psi}_{v}: \mathbb{Q}\left[M_{\beta(\sigma)}\right]\left[\operatorname{deg}\left(y^{(v, \sigma)}\right)\right] \rightarrow y^{(v, \sigma)} \cdot \mathbb{Q}\left[M_{\beta}\right]$ be the morphism given by $y^{\widetilde{b}_{i}} \mapsto y^{(v, \sigma)} \cdot y^{b_{i}}$. Using similar arguments as in Proposition 4.7 of [Jiang], we find that the ideal $\operatorname{Cir}\left(\Delta_{\beta(\sigma)}\right)$ goes to the ideal $y^{(v, \sigma)} \cdot \operatorname{Cir}\left(\Delta_{\beta}\right)$, so we have the morphism $\Psi_{v}$ such that $\Psi_{v}\left(\left[y^{\widetilde{b}_{i}}\right]\right)=\left[y^{(v, \sigma)} \cdot y^{b_{i}}\right]$.

Conversely, for $(v, \sigma) \in \operatorname{Box}\left(\Delta_{\beta}\right)$, since $\sigma$ is simplicial, for $\rho_{i} \subset \sigma$ we can choose $\theta_{i} \in$ $\operatorname{Hom}_{\mathbb{Z}}(N, \mathbb{Q})$ such that $\theta_{i}\left(b_{i}\right)=1$ and $\theta_{i}\left(b_{i^{\prime}}\right)=0$ for $b_{i^{\prime}} \neq b_{i} \in \sigma$. We consider the following morphism $p: \mathbb{Q}\left[y^{b_{1}}, \ldots, y^{b_{m}}\right] \rightarrow \mathbb{Q}\left[y^{\widetilde{b}_{1}}, \ldots, y^{\widetilde{b}_{l}}\right]$ given by:

$$
y^{b_{i}} \longmapsto \begin{cases}y^{\widetilde{b}_{i}} & \text { if } \rho_{i} \subseteq \operatorname{link}(\sigma), \\ -\sum_{j=1}^{l} \theta_{i}\left(b_{j}\right) y^{\widetilde{b}_{j}} & \text { if } \rho_{i} \subseteq \sigma, \\ 0 & \text { if } \rho_{i} \nsubseteq \sigma \cup \operatorname{link}(\sigma) .\end{cases}
$$

Again by Lemma 5.7, the ideal $I_{M_{\beta}}$ is mapped by $p$ to the ideal $I_{M_{\beta(\sigma)}}$. Then $p$ induces a surjective $\operatorname{map} \mathbb{Q}\left[M_{\beta}\right] \rightarrow \mathbb{Q}\left[M_{\beta(\sigma)}\right]$ and a surjective map $\widetilde{\Phi}_{v}: y^{(v, \sigma)} \cdot \mathbb{Q}\left[M_{\beta}\right] \rightarrow \mathbb{Q}\left[M_{\beta(\sigma)}\right]\left[\operatorname{deg}\left(y^{(v, \sigma)}\right)\right]$. Using the same computation as in Proposition 4.7 in [Jiang], the relations $y^{(v, \sigma)} \cdot \operatorname{Cir}\left(\Delta_{\beta}\right)$ is seen to go to $\operatorname{Cir}\left(\Delta_{\beta(\sigma)}\right)$. This yields another morphism

$$
\Phi_{v}: \frac{y^{(v, \sigma)} \cdot \mathbb{Q}\left[M_{\beta}\right]}{y^{(v, \sigma)} \cdot \operatorname{Cir}\left(\Delta_{\beta}\right)} \longrightarrow \frac{\mathbb{Q}\left[M_{\beta(\sigma)}\right]}{\operatorname{Cir}\left(\Delta_{\beta(\sigma)}\right)}\left[\operatorname{deg}\left(y^{(v, \sigma)}\right)\right]
$$

so that $\Phi_{v} \Psi_{v}=1, \Psi_{v} \Phi_{v}=1$. So $\Psi_{v}$ is an isomorphism. We conclude by Lemma 5.5,

5.2. The orbifold product. In this section we compute the orbifold cup product. First for any $\left(v_{1}, \sigma_{1}\right),\left(v_{2}, \sigma_{2}\right) \in \operatorname{Box}\left(\Delta_{\beta}\right)$, we have the following lemma:

Lemma 5.9. If $\sigma_{1} \cup \sigma_{2}$ is a cone in the multi-fan $\Delta_{\beta}$, there exists a unique $\left(v_{3}, \sigma_{3}\right) \in \operatorname{Box}\left(\Delta_{\beta}\right)$ such that $\sigma_{1} \cup \sigma_{2} \cup \sigma_{3}$ is a cone in the multi-fan $\Delta_{\beta}$ and $v_{1}+v_{2}+v_{3}$ has no fractional part.

Proof. Let $v_{3}=\left\lceil v_{1}+v_{2}\right\rceil_{\sigma_{1} \cup \sigma_{2}}-v_{1}-v_{2}$ and $\sigma_{3}$ the minimal cone in $\sigma_{1} \cup \sigma_{2}$ containing $v_{3}$. Then $\left(v_{3}, \sigma_{3}\right)$ satisfies the conditions of the Lemma.

The notation $\left(v_{1}, \sigma_{1}\right)+\left(v_{2}, \sigma_{2}\right)+\left(v_{3}, \sigma_{3}\right) \equiv 0$ means that the triple $\left(\left(v_{1}, \sigma_{1}\right),\left(v_{2}, \sigma_{2}\right),\left(v_{3}, \sigma_{3}\right)\right)$ satisfies the conditions in Lemma 5.9 .

By [CR2, the 3-twisted sector $\mathcal{M}(\mathcal{A})_{\left(g_{1}, g_{2}, g_{3}\right)}$ is the moduli space of 3 -pointed genus 0 degree 0 orbifold stable maps to $\mathcal{M}(\mathcal{A})$. Let $\mathbb{P}^{1}(0,1, \infty)$ be a genus 0 twisted curve with 
stacky structures possibly at $0,1, \infty$. Consider a constant map $f: \mathbb{P}^{1}(0,1, \infty) \rightarrow \mathcal{M}(\mathcal{A})$ with image $x \in \mathcal{M}(\mathcal{A})$. This induces a morphism

$$
\rho: \pi_{1}^{\text {orb }}\left(\mathbb{P}^{1}(0,1, \infty)\right) \rightarrow G_{x}
$$

where $G_{x}$ is the local group of the point $x$. Let $\gamma_{i}$ be generators of $\pi_{1}^{\text {orb }}\left(\mathbb{P}^{1}(0,1, \infty)\right)$ and $g_{i}:=\rho\left(\gamma_{i}\right)$. The $g_{i}$ fixes the point $x$. By Proposition 4.6, $g_{i}$ corresponds to an element $\left(v_{i}, \sigma_{i}\right) \in \operatorname{Box}\left(\Delta_{\beta}\right)$. An argument similar to that in Proposition 6.1 in [BCS] shows that 3 -twisted sectors of the hypertoric DM stack $\mathcal{M}(\mathcal{A})$ are given by

$$
\coprod_{\left(\left(v_{1}, \sigma_{1}\right),\left(v_{2}, \sigma_{2}\right),\left(v_{3}, \sigma_{3}\right)\right) \in \operatorname{Box}\left(\Delta_{\beta}\right)^{3},\left(v_{1}, \sigma_{1}\right)+\left(v_{2}, \sigma_{2}\right)+\left(v_{3}, \sigma_{3}\right) \equiv 0} \mathcal{M}\left(\mathcal{A}\left(\sigma_{123}\right)\right),
$$

where $\sigma_{123}$ is the cone in $\Delta_{\beta}$ satisfying $v_{1}+v_{2}+v_{3}=\sum_{\rho_{i} \subset \sigma_{123}} a_{i} b_{i}, a_{i}=1,2$. Let $e v_{i}$ : $\mathcal{M}\left(\mathcal{A}\left(\sigma_{123}\right)\right) \rightarrow \mathcal{M}\left(\mathcal{A}\left(\sigma_{i}\right)\right)$ be the evaluation map. We have the obstruction bundle (see CR1]) $\operatorname{Ob}_{\left(v_{1}, v_{2}, v_{3}\right)}$ over the 3-twisted sector $\mathcal{M}\left(\mathcal{A}\left(\sigma_{123}\right)\right)$,

$$
O b_{\left(v_{1}, v_{2}, v_{3}\right)}=\left(e^{*} T(\mathcal{M}(\mathcal{A})) \otimes H^{1}\left(C, \mathcal{O}_{C}\right)\right)^{H}
$$

where $e: \mathcal{M}\left(\mathcal{A}\left(\sigma_{123}\right)\right) \rightarrow \mathcal{M}(\mathcal{A})$ is the embedding, $C \rightarrow \mathbb{P}^{1}$ is the $H$-covering branching over three marked points $\{0,1, \infty\} \subset \mathbb{P}^{1}$, and $H$ is the group generated by $v_{1}, v_{2}, v_{3}$. Let $(v, \sigma) \in \operatorname{Box}\left(\Delta_{\beta}\right)$, say $v \in N(\tau)$ for some top dimensional cone $\tau$. Let $(\check{v}, \sigma) \in \operatorname{Box}\left(\Delta_{\beta}\right)$ be the inverse of $v$ as an element in the group $N(\tau)$. Equivalently, if $\bar{v}=\sum_{\rho_{i} \subseteq \sigma} \alpha_{i} \bar{b}_{i}$ for $0<\alpha_{i}<1$, then $\check{\bar{v}}=\sum_{\rho_{i} \subseteq \sigma}\left(1-\alpha_{i}\right) \bar{b}_{i}$.

Lemma 5.10. Let $\left(v_{1}, \sigma_{1}\right),\left(v_{2}, \sigma_{2}\right),\left(v_{3}, \sigma_{3}\right) \in \operatorname{Box}\left(\Delta_{\beta}\right)$ such that $v_{1}+v_{2}+v_{3} \equiv 0$. Then if $\bar{v}_{1}+\bar{v}_{2}+\bar{v}_{3}=\sum_{\rho_{i} \subseteq \sigma_{123}} a_{i} \bar{b}_{i}, \check{\bar{v}}_{1}+\check{\bar{v}}_{2}+\check{\bar{v}}_{3}=\sum_{\rho_{i} \subseteq \sigma_{123}} c_{i} \bar{b}_{i}$, where $a_{i}, c_{i}=1$ or 2 , then $a_{i}+c_{i}=2$ or 3 .

ProOF. Let $\bar{v}_{i}=\sum_{\rho_{j} \subseteq \sigma_{i}} \alpha_{j}^{i} \bar{b}_{j}$, with $0<\alpha_{j}^{i}<1$ and $i=1,2,3$. Then $\check{\bar{v}}_{i}=\sum_{\rho_{j} \subseteq \sigma_{i}}\left(1-\alpha_{j}^{i}\right) \bar{b}_{j}$. From the condition we have $\alpha_{j}^{1}+\alpha_{j}^{2}+\alpha_{j}^{3}=a_{j}=1$ or 2 and $\left(1-\alpha_{j}^{1}\right)+\left(1-\alpha_{j}^{2}\right)+\left(1-\alpha_{j}^{3}\right)=$ $c_{j}=2$ or 1 . So if $\rho_{j}$ belongs to $\sigma_{1}, \sigma_{2}$ and $\sigma_{3}$, then $\alpha_{j}^{1}, \alpha_{j}^{2}, \alpha_{j}^{3}$ exist and if $a_{j}=1$ or 2 , then $c_{j}=2$ or 1 . If $\rho_{j}$ belongs to $\sigma_{1}, \sigma_{2}$, but not $\sigma_{3}$, then $\alpha_{j}^{3}$ doesn't exist and $\alpha_{j}^{1}+\alpha_{j}^{2}=a_{j}=1$, $\left(1-\alpha_{j}^{1}\right)+\left(1-\alpha_{j}^{2}\right)=c_{j}=1$. The cases that $\rho_{j}$ belongs to $\sigma_{1}, \sigma_{3}$ but not $\sigma_{2}$, to $\sigma_{2}, \sigma_{3}$ but not $\sigma_{1}$ are similar. We omit them.

The stack $\mathcal{M}(\mathcal{A})$ is an abelian DM stack, i.e. the local groups are all abelian groups. For any 3-twisted sector $\mathcal{M}\left(\mathcal{A}\left(\sigma_{123}\right)\right)$, the normal bundle $N\left(\mathcal{M}\left(\mathcal{A}\left(\sigma_{123}\right)\right) / \mathcal{M}(\mathcal{A})\right)$ can be split into the direct sum of some line bundles under the group action. It follows from the definition that if $\bar{v}=\sum_{\rho_{i} \subseteq \sigma_{123}} \alpha_{i} \bar{b}_{i}$, then the action of $v$ on the normal bundle $N\left(\mathcal{M}\left(\mathcal{A}\left(\sigma_{123}\right)\right) / \mathcal{M}(\mathcal{A})\right)$ is given by the diagonal matrix $\operatorname{diag}\left(\alpha_{i}, 1-\alpha_{i}\right)$. A general result in [CH] and [JKK] about the obstruction bundle and Lemma 5.10 imply the following Proposition.

Proposition 5.11. Let $\mathcal{M}(\mathcal{A})_{\left(v_{1}, v_{2}, v_{3}\right)}=\mathcal{M}\left(\mathcal{A}\left(\sigma_{123}\right)\right)$ be a 3-twisted sector of the stack $\mathcal{M}(\mathcal{A})$ such that $v_{1}, v_{2}, v_{3} \neq 0$. Then the Euler class of the obstruction bundle $\mathrm{Ob}_{\left(v_{1}, v_{2}, v_{3}\right)}$ on $\mathcal{M}\left(\mathcal{A}\left(\sigma_{123}\right)\right)$ is

$$
\left.\left.\prod_{a_{i}=2} c_{1}\left(L_{i}\right)\right|_{\mathcal{M}\left(\mathcal{A}\left(\sigma_{123}\right)\right)} \cdot \prod_{a_{i}=1, \alpha_{j}^{1}, \alpha_{j}^{2}, \alpha_{j}^{3}} c_{\text {exist }}\left(L_{i}^{-1}\right)\right|_{\mathcal{M}\left(\mathcal{A}\left(\sigma_{123}\right)\right)},
$$

where $L_{i}$ is the line bundle over $\mathcal{M}(\mathcal{A})$ defined in Definition 5.1. 
To prove the main theorem, we introduce two Lemmas. For any two pairs $\left(c_{1}, \sigma_{1}\right),\left(c_{2}, \sigma_{2}\right) \in$ $N^{\Delta_{\beta}}$, there exist two unique elements $\left(v_{1}, \tau_{1}\right),\left(v_{2}, \tau_{2}\right) \in \operatorname{Box}\left(\Delta_{\beta}\right)$ such that $\tau_{1} \subseteq \sigma_{1}, \tau_{2} \subseteq \sigma_{2}$ and $c_{1}=v_{1}+\sum_{\rho_{i} \subseteq \sigma_{1}} m_{i} b_{i}, c_{2}=v_{2}+\sum_{\rho_{i} \subseteq \sigma_{2}} n_{i} b_{i}$, where $m_{i}, n_{i}$ are nonnegative integers. Let $\left(v_{3}, \sigma_{3}\right)$ be the unique element in $\operatorname{Box}\left(\Delta_{\beta}\right)$ such that $v_{1}+v_{2}+v_{3} \equiv 0$ in the local group given by $\sigma_{1} \cup \sigma_{2}$. Let $\bar{v}_{i}=\sum_{\rho_{j} \subset \sigma_{i}} \alpha_{j}^{i} \bar{b}_{j}$, with $0<\alpha_{j}^{i}<1$ and $i=1,2,3$. The existence of $\alpha_{j}^{1}, \alpha_{j}^{2}, \alpha_{j}^{3}$ means that $\rho_{j}$ belongs to $\sigma_{1}, \sigma_{2}, \sigma_{3}$. Let $\sigma_{123}$ be the cone in $\Delta_{\beta}$ such that $\bar{v}_{1}+\bar{v}_{2}+\bar{v}_{3}=\sum_{\rho_{i} \subseteq \sigma_{123}} a_{i} \bar{b}_{i}$, with $a_{i}=1$ or 2 . Let $I$ be the set of $i$ such that $a_{i}=1$ and $\alpha_{j}^{1}, \alpha_{j}^{2}, \alpha_{j}^{3}$ exist, $J$ the set of $j$ such that $\rho_{j}$ belongs to $\sigma_{123}$ but not to $\sigma_{3}$. We have the following lemma for the ceiling functions:

Lemma 5.12. $\left\lceil c_{1}\right\rceil_{\sigma_{1}}+\left\lceil c_{2}\right\rceil_{\sigma_{2}}-\left\lceil c_{1}+c_{2}\right\rceil_{\sigma_{1} \cup \sigma_{2}}=\left\lceil v_{1}\right\rceil_{\tau_{1}}+\left\lceil v_{2}\right\rceil_{\tau_{2}}-\left\lceil v_{1}+v_{2}\right\rceil_{\tau_{1} \cup \tau_{2}}$.

ProOF. By the definition of ceiling functions, we have $\left\lceil c_{1}\right\rceil_{\sigma_{1}}=\left\lceil v_{1}\right\rceil_{\tau_{1}}+\sum_{\rho_{i} \subseteq \sigma_{1}} m_{i} b_{i}$ and $\left\lceil c_{2}\right\rceil_{\sigma_{2}}=\left\lceil v_{2}\right\rceil_{\tau_{2}}+\sum_{\rho_{i} \subseteq \sigma_{2}} n_{i} b_{i}$. The Lemma follows.

Lemma 5.13. If $\sigma_{1} \cup \sigma_{2}$ is a cone in $\Delta_{\beta}$ for the two pairs $\left(c_{1}, \sigma_{1}\right),\left(c_{2}, \sigma_{2}\right)$, then the product $y^{\left(c_{1}, \sigma_{1}\right)} \cdot y^{\left(c_{2}, \sigma_{2}\right)}$ in (1.1) can be given by

$$
\begin{cases}(-1)^{|I|+|J|} y^{\left(c_{1}+c_{2}+\sum_{i \in I} b_{i}+\sum_{i \in J} b_{i}, \sigma_{1} \cup \sigma_{2}\right)} & \text { if } \bar{v}_{1}, \bar{v}_{2} \neq 0 \text { and } \bar{v}_{1} \neq \check{v}_{2}, \\ (-1)^{|J|} y^{\left(c_{1}+c_{2}+\sum_{i \in J} b_{i}, \sigma_{1} \cup \sigma_{2}\right)} & \text { if } \bar{v}_{1}, \bar{v}_{2} \neq 0 \text { and } \bar{v}_{1}=\check{\bar{v}}_{2}, \\ y^{\left(c_{1}+c_{2}, \sigma_{1} \cup \sigma_{2}\right)} & \text { if } \bar{v}_{1} \text { or } \bar{v}_{2}=0 .\end{cases}
$$

Proof. First for a fixed ray $\rho_{i}$ and $0<\alpha_{1}, \alpha_{2}<1$, by the definition of ceiling functions, we find that

$$
\left\lceil\alpha_{1} b_{i}\right\rceil_{\rho_{i}}+\left\lceil\alpha_{2} b_{i}\right\rceil_{\rho_{i}}-\left\lceil\alpha_{1} b_{i}+\alpha_{2} b_{i}\right\rceil_{\rho_{i}}= \begin{cases}0 & \text { if } \alpha_{1}+\alpha_{2}>1 \\ b_{i} & \text { if } \alpha_{1}+\alpha_{2} \leq 1\end{cases}
$$

Since $\epsilon\left(c_{1}, c_{2}\right)=\left\lceil c_{1}\right\rceil_{\sigma_{1}}+\left\lceil c_{2}\right\rceil_{\sigma_{2}}-\left\lceil c_{1}+c_{2}\right\rceil_{\sigma_{1} \cup \sigma_{2}}$, by Lemma 5.12, we need to check that

$$
\left\lceil v_{1}\right\rceil_{\tau_{1}}+\left\lceil v_{2}\right\rceil_{\tau_{2}}-\left\lceil v_{1}+v_{2}\right\rceil_{\tau_{1} \cup \tau_{2}}= \begin{cases}\sum_{i \in I} b_{i}+\sum_{i \in J} b_{i} & \text { if } \bar{v}_{1}, \bar{v}_{2} \neq 0 \text { and } \bar{v}_{1} \neq \check{v}_{2}, \\ \sum_{i \in J} b_{i} & \text { if } \bar{v}_{1}, \bar{v}_{2} \neq 0 \text { and } \bar{v}_{1}=\check{\bar{v}}_{2}, \\ 0 & \text { if } \bar{v}_{1} \text { or } \bar{v}_{2}=0 .\end{cases}
$$

This can be easily proven using (5.4) and Lemma 5.10,

5.3. Proof of Theorem 1.1. By Proposition 5.8, it remains to prove that the orbifold cup product is the same as the product in the ring $\mathbb{Q}\left[\Delta_{\beta}\right]$. By Lemma 5.13, we need to prove that the orbifold cup product is the same as the product in (5.3). It suffices to consider the canonical generators $y^{b_{i}}, y^{(v, \sigma)}$ for $(v, \sigma) \in \operatorname{Box}\left(\Delta_{\beta}\right)$.

Consider $y^{(v, \sigma)} \cup_{\text {orb }} y^{b_{i}}$ with $(v, \sigma) \in \operatorname{Box}\left(\Delta_{\beta}\right)$. The element $(v, \sigma)$ determines a twisted sector $\mathcal{M}(\mathcal{A}(\sigma))$. The corresponding twisted sector to $b_{i}$ is the whole hypertoric stack $\mathcal{M}(\mathcal{A})$. It is easy to see that the 3 -twisted sector relevant to this product is $\mathcal{M}(\mathcal{A})_{\left(v, 1, v^{-1}\right)} \cong \mathcal{M}(\mathcal{A}(\sigma))$, where $v^{-1}$ denotes the inverse of $v$ in the local group. It follows from the dimension formula in [CR1] that the obstruction bundle over $\mathcal{M}(\mathcal{A})_{\left(v, 1, v^{-1}\right)}$ has rank zero. It is immediate from definition that $y^{(v, \sigma)} \cup_{\text {orb }} y^{b_{i}}=y^{\left(v+b_{i}, \sigma \cup \rho_{i}\right)}$ if there is a cone in $\Delta_{\beta}$ containing $\bar{v}, \bar{b}_{i}$. This is the third case in (5.3). 
Now consider $y^{\left(v_{1}, \sigma_{1}\right)} \cup_{\text {orb }} y^{\left(v_{2}, \sigma_{2}\right)}$, where $\left(v_{1}, \sigma_{1}\right),\left(v_{2}, \sigma_{2}\right) \in \operatorname{Box}\left(\Delta_{\beta}\right)$. By (15.1), we see that if $\sigma_{1} \cup \sigma_{2}$ is not a cone in $\Delta_{\beta}$, then there is no 3-twisted sector corresponding to the elements $v_{1}, v_{2}$. Thus the product is zero by definition. On the other hand, by definition of the ring $\mathbb{Q}\left[\Delta_{\beta}\right]$, we have $y^{\left(v_{1}, \sigma_{1}\right)} \cdot y^{\left(v_{2}, \sigma_{2}\right)}=0$. So $y^{\left(v_{1}, \sigma_{1}\right)} \cup_{\text {orb }} y^{\left(v_{2}, \sigma_{2}\right)}=y^{\left(v_{1}, \sigma_{1}\right)} \cdot y^{\left(v_{2}, \sigma_{2}\right)}$. If $\sigma_{1} \cup \sigma_{2}$ is a cone in $\Delta_{\beta}$, let $\left(v_{3}, \sigma_{3}\right) \in \operatorname{Box}\left(\Delta_{\beta}\right)$ such that $\bar{v}_{3} \in \sigma_{123}$ and $v_{1} v_{2} v_{3}=1$ in the local group. Then we have the 3 -twisted sector $\mathcal{M}\left(\mathcal{A}\left(\sigma_{123}\right)\right)$. Let $e v_{i}: \mathcal{M}\left(\mathcal{A}\left(\sigma_{123}\right)\right) \rightarrow \mathcal{M}\left(\mathcal{A}\left(\sigma_{i}\right)\right)$ be the evaluation maps. The element $y^{(v, \sigma)}$ is the class 1 in the cohomology of the twisted sector $\mathcal{M}(\mathcal{A}(\sigma))$. From the definition of orbifold cup product [CR1], [GG], we have:

$$
y^{\left(v_{1}, \sigma_{1}\right)} \cup_{o r b} y^{\left(v_{2}, \sigma_{2}\right)}=\left(e \breve{v}_{3}\right)_{*}\left(e v_{1}^{*} y^{\left(v_{1}, \sigma_{1}\right)} \cdot e v_{2}^{*} y^{\left(v_{2}, \sigma_{2}\right)} \cdot e\left(O b_{\left(v_{1}, v_{2}, v_{3}\right)}\right)\right)
$$

where $e \breve{v v}_{3}=\mathcal{I} \circ e v_{3}: \mathcal{M}\left(\mathcal{A}\left(\sigma_{123}\right)\right) \rightarrow \mathcal{M}(\mathcal{A})_{\left(\check{v}_{3}\right)}$ is the composite of $e v_{3}$ and the natural involution $\mathcal{I}: \mathcal{M}(\mathcal{A})_{\left(v_{3}\right)} \rightarrow \mathcal{M}(\mathcal{A})_{\left(\check{v}_{3}\right)}$. Let $\bar{v}_{i}=\sum_{\rho_{j} \subseteq \sigma_{i}} \alpha_{j}^{i} \bar{b}_{j}$, with $0<\alpha_{j}^{i}<1$ and $i=1,2,3$. Let $I$ denote the set of $i$ such that $a_{i}=1$ and $\alpha_{j}^{1}, \alpha_{j}^{2}, \alpha_{j}^{3}$ exist, $J$ the set of $j$ such that $\rho_{i}$ belongs to $\sigma_{123}$, but not belong to $\sigma_{3}$.

If some $\bar{v}_{i}=0$, for example, $\bar{v}_{1}=0$, then $v_{1}$ is a torsion element in $N$ which means that the action of $v_{1}$ is trivial on the hypertoric DM stack. Then the 3-twisted sector corresponding to $v_{1}, v_{2}$ is isomorphic to the twisted sector $\mathcal{M}\left(\mathcal{A}\left(\sigma_{2}\right)\right)$ and the obstruction bundle over $\mathcal{M}\left(\mathcal{A}\left(\sigma_{2}\right)\right)$ is zero by [CR1]. In this case the set $I$ and $J$ are all empty. So $y^{\left(v, \sigma_{1}\right)} \cup_{\text {orb }} y^{\left(v, \sigma_{2}\right)}=y^{\left(v_{1}+v_{2}, \sigma_{1} \cup \sigma_{2}\right)}$. This is again the third case in (5.3).

Now we assume that $\bar{v}_{1}, \bar{v}_{2} \neq 0$. If $\bar{v}_{1}=\check{\bar{v}}_{2}$, then $\bar{v}_{3}=0, \sigma_{123}=\sigma_{1}$ and $v_{1}+v_{2}=\sum_{\rho_{j} \subseteq \sigma_{1}} b_{j}$. So the 3-twisted sector corresponding to $v_{1}, v_{2}$ is isomorphic to the twisted sector $\mathcal{M}\left(\mathcal{A}\left(\sigma_{1}\right)\right)$ and the obstruction bundle over $\mathcal{M}\left(\mathcal{A}\left(\sigma_{1}\right)\right)$ is zero by [CR1]. The set $J$ is the set $j$ such that $\rho_{j} \subseteq \sigma_{1}$. So we have

$$
\begin{aligned}
y^{\left(v_{1}, \sigma_{1}\right)} \cup_{\text {orb }} y^{\left(v_{2}, \sigma_{2}\right)} & =y^{0} \cdot \prod_{i \in J} y^{b_{i}} \cdot \prod_{i \in J}\left(-y^{b_{i}}\right) \\
& =(-1)^{|J|} \cdot y^{\left(v_{1}+v_{2}+\sum_{i \in J} b_{i}, \sigma_{1} \cup \sigma_{2}\right)},
\end{aligned}
$$

which is the second case in (5.3).

If $\bar{v}_{1} \neq \check{\bar{v}}_{2}$, then $\bar{v}_{3} \neq 0$ and the obstruction bundle over the 3 -twisted sector $\mathcal{M}\left(\mathcal{A}\left(\sigma_{123}\right)\right)$ is given by Proposition 5.11. So we have:

$$
y^{\left(v_{1}, \sigma_{1}\right)} \cup_{\text {orb }} y^{\left(v_{2}, \sigma_{2}\right)}=y^{\left(\check{v}_{3}, \sigma_{3}\right)} \cdot \prod_{a_{i}=2} y^{b_{i}} \cdot \prod_{i \in J} y^{b_{i}} \cdot \prod_{i \in I}\left(-y^{b_{i}}\right) \cdot \prod_{i \in J}\left(-y^{b_{i}}\right) .
$$

Since $\check{v}_{3}+\sum_{a_{i}=2} b_{i}+\sum_{i \in J} b_{i}=v_{1}+v_{2}$, we have

$$
\begin{aligned}
y^{\left(v_{1}, \sigma_{1}\right)} \cup_{\text {orb }} y^{\left(v_{2}, \sigma_{2}\right)} & =(-1)^{|I|+|J|} \cdot y^{\left(v_{1}+v_{2}, \sigma_{1} \cup \sigma_{2}\right)} \cdot \prod_{i \in I} y^{b_{i}} \cdot \prod_{i \in J} y^{b_{i}} \\
& =(-1)^{|I|+|J|} \cdot y^{\left(v_{1}+v_{2}+\sum_{i \in I} b_{i}+\sum_{i \in J} b_{i}, \sigma_{1} \cup \sigma_{2}\right)},
\end{aligned}
$$

which is the first case in (5.3).

\section{Applications}

In this section we compute some examples of the orbifold Chow rings of hypertoric DM stacks. In particular, we relate the hypertoric stack to crepant resolutions. 
Let $N=\mathbb{Z}$ and $\Sigma$ the fan of projective line $\mathbb{P}^{1}$ generated by $\{(1),(-1)\}$. Let $\beta: \mathbb{Z}^{n} \rightarrow N$ be the map given by $b_{1}=(1), b_{2}=(-1)$ and $b_{i}=(1)$ for $i \geq 2$. Consider the following exact sequences

$$
\begin{aligned}
& 0 \longrightarrow \mathbb{Z}^{n-1} \longrightarrow \mathbb{Z}^{n} \stackrel{\beta}{\longrightarrow} N \longrightarrow 0 \longrightarrow 0, \\
& 0 \longrightarrow \mathbb{Z} \longrightarrow \mathbb{Z}^{n} \stackrel{\beta^{\vee}}{\longrightarrow} \mathbb{Z}^{n-1} \longrightarrow 0 \longrightarrow 0,
\end{aligned}
$$

where the Gale dual $\beta^{\vee}$ is given by the column vectors of the matrix

$$
A=\left[\begin{array}{cccccc}
1 & 1 & 0 & 0 & \cdots & 0 \\
1 & 0 & -1 & 0 & \cdots & 0 \\
1 & 0 & 0 & -1 & \cdots & 0 \\
\vdots & \vdots & \vdots & \vdots & \ddots & \vdots \\
1 & 0 & 0 & 0 & \cdots & -1
\end{array}\right]
$$

Note that $A$ is unimodular in the sense of $[\mathrm{HS}]$. Taking $\operatorname{Hom}_{\mathbb{Z}}\left(-, \mathbb{C}^{\times}\right)$yields

$$
1 \longrightarrow\left(\mathbb{C}^{\times}\right)^{n-1} \stackrel{\alpha}{\longrightarrow}\left(\mathbb{C}^{\times}\right)^{n} \longrightarrow \mathbb{C}^{\times} \longrightarrow 1 .
$$

So $G=\left(\mathbb{C}^{\times}\right)^{n-1}$. Choose $\theta=(1,1, \cdots, 1)$ in $\mathbb{Z}^{n-1}$, then it is a generic element. The extended stacky fan $\Sigma=(N, \Sigma, \beta)$ is induced from the stacky hyperplane arrangement $\mathcal{A}=(N, \beta, \theta)$, where $\mathcal{H}$ is the hyperplane arrangement whose normal fan is $\Sigma$. It is easy to see that the toric DM stack is the projective line $\mathbb{P}^{1}$. The hypertoric DM stack is the crepant resolution of the Gorenstein orbifold $\left[\mathbb{C}^{2} / \mathbb{Z}_{n}\right]$. To see this, from the construction of hypertoric DM stack, we have:

$$
1 \longrightarrow\left(\mathbb{C}^{\times}\right)^{n-1} \stackrel{\alpha^{L}}{\longrightarrow}\left(\mathbb{C}^{\times}\right)^{2 n} \longrightarrow\left(\mathbb{C}^{\times}\right)^{n+1} \longrightarrow 1,
$$

where $\alpha^{L}$ is given by the matrix $\left[\beta^{\vee},-\beta^{\vee}\right]$. Let $\mathbb{C}\left[z_{1}, \ldots, z_{n}, w_{1}, \ldots, w_{n}\right]$ be the coordinate ring of $\mathbb{C}^{2 n}$. So the ideal $I_{\beta^{\vee}}$ in (2.9) is generated by the following equations:

$$
\left\{\begin{array}{l}
z_{1} w_{1}+z_{2} w_{2}=0, \\
z_{1} w_{1}-z_{3} w_{3}=0, \\
\cdots \ldots \ldots \ldots \\
z_{1} w_{1}-z_{n} w_{n}=0 .
\end{array}\right.
$$

Hence $Y$ is the subvariety of $\mathbb{C}^{2 n}-V\left(\mathcal{I}_{\theta}\right)$ determined by the above ideal. The action of $G$ on $Y$ is through the map $\alpha^{L}$ in (6.1). The hypertoric DM stack associated to $\mathcal{A}$ is $\mathcal{M}(\mathcal{A})=[Y / G]$. From Proposition 3.3, the hypertoric DM stack is independent to the coorientations of the hyperplanes. This means that we can give the stacky hyperplane arrangement $\mathcal{A}$ as follows. Let $b_{i}=1$ for $1 \leq i \leq n$. Then the Gale dual map $\beta^{\vee}: \mathbb{Z}^{n} \rightarrow \mathbb{Z}^{n-1}$ is given by the matrix

$$
A=\left[\begin{array}{cccccc}
1 & -1 & 0 & 0 & \ldots & 0 \\
0 & 1 & -1 & 0 & \ldots & 0 \\
0 & 0 & 1 & -1 & \ldots & 0 \\
\vdots & \vdots & \ddots & \ddots & \ddots & \vdots \\
0 & 0 & 0 & \cdots & 1 & -1
\end{array}\right]
$$

which is exactly the matrix in Lemma 10.2 in [HS], from which it follows that the coarse moduli space $Y\left(\beta^{\vee}, \theta\right)$ of $\mathcal{M}(\mathcal{A})=[Y / G]$ is the crepant resolution of the Gorenstein orbifold 
$\left[\mathbb{C}^{2} / \mathbb{Z}_{n}\right]$. The core of the hypertoric DM stack $\mathcal{M}(\mathcal{A})$ is a chain of $n-1$ copies of $\mathbb{P}^{1}$ with normal crossing divisors corresponding to the multi-fan $\Delta_{\beta}$.

Remark 6.1. This is an example of [Kro], in which it is shown that the minimal resolution of a surface singularity of ADE type can be constructed as a hyperkähler quotient.

The $\mathbb{Z}_{n}$-action defining the Gorenstein orbifold $\left[\mathbb{C}^{2} / \mathbb{Z}_{n}\right]$ is given by $\lambda(x, y)=\left(\lambda x, \lambda^{-1} y\right)$ for $\lambda \in \mathbb{Z}_{n}$. There are $n-1$ twisted sectors each of which is isomorphic to $\mathcal{B} \mathbb{Z}_{n}$ with age 1 . There are only dimension zero cohomology on the untwisted sector and twisted sectors. So we prove the following Proposition:

Proposition 6.2. The orbifold Chow ring $A_{\text {orb }}^{*}\left(\left[\mathbb{C}^{2} / \mathbb{Z}_{n}\right]\right)$ of $\left[\mathbb{C}^{2} / \mathbb{Z}_{n}\right]$ is isomorphic to the ring

$$
\frac{\mathbb{C}\left[x_{1}, \cdots, x_{n-1}\right]}{\left\{x_{i} x_{j}: 1 \leq i, j \leq n-1\right\}} .
$$

Since the crepant resolution is a manifold, the orbifold Chow ring is the ordinary Chow ring. By Theorem 1.1, we have

Proposition 6.3. The Chow ring of $\mathcal{M}(\mathcal{A})$ is isomorphic to the ring

$$
\frac{\mathbb{C}\left[y_{1}, \cdots, y_{n-1}\right]}{\left\{y_{i} y_{j}: 1 \leq i, j \leq n-1\right\}}
$$

which is isomorphic to the orbifold cohomology ring of the Gorenstein orbifold $\left[\mathbb{C}^{2} / \mathbb{Z}_{n}\right]$.

Proof. By Theorem 1.1, the Chow ring of $\mathcal{M}(\mathcal{A})$ is isomorphic to the ring:

$$
\frac{\mathbb{C}\left[y_{1}, \cdots, y_{n}\right]}{\left\{y_{1}-y_{n}+y_{3}+\cdots+y_{n-1}, y_{i} y_{j}: 1 \leq i, j \leq n-1\right\}}
$$

which we can easily check that this ring is isomorphic to the orbifold cohomology ring of $\left[\mathbb{C}^{2} / \mathbb{Z}_{n}\right]$ in Proposition 6.2.

Y. Ruan $[\mathrm{R}]$ conjectured that, among other things, the orbifold cohomology ring of a hyperkähler orbifold is isomorphic to the ordinary cohomology ring of a hyperkähler resolution (which is crepant). For the orbifold $\left[\mathbb{C}^{2} / \mathbb{Z}_{n}\right]$, the crepant resolution $Y\left(\beta^{\vee}, \theta\right)$ is smooth, we have that $\mathcal{M}(\mathcal{A}) \cong Y\left(\beta^{\vee}, \theta\right)$. From Proposition 6.3, the conjecture is true.

A conjecture equating Gromov-Witten theories of an orbifold and its crepant resolutions, as proposed in $[\mathrm{BG}]$, is recently proven in genus 0 for $\left[\mathbb{C}^{2} / \mathbb{Z}_{3}\right]$, see $[\mathrm{BGP}$. The comparison of two Gromov-Witten theories requires certain change of variables. For $\left[\mathbb{C}^{2} / \mathbb{Z}_{3}\right]$ case, see $\left[\mathrm{BGP}\right.$. For $\left[\mathbb{C}^{2} / \mathbb{Z}_{4}\right]$ case the following change of variables is found in $[\mathrm{BJ}]$ :

$$
\left\{\begin{array}{l}
y_{1}=\frac{1}{4}\left(\sqrt{2} x_{1}+2 i x_{2}-\sqrt{2} x_{3}\right) \\
y_{2}=\frac{1}{4}\left(\sqrt{2} i x_{1}-2 i x_{2}+\sqrt{2} i x_{3}\right) \\
y_{3}=\frac{1}{4}\left(-\sqrt{2} x_{1}+2 i x_{2}+\sqrt{2} x_{3}\right) .
\end{array}\right.
$$

Under this change of variables, the genus zero Gromov-Witten potential of the crepant resolution is seen to coincide with the genus zero orbifold Gromov-Witten potential of the orbifold $\left[\mathbb{C}^{2} / \mathbb{Z}_{4}\right]$, see $[\mathrm{BJ}]$.

For a toric orbifold, it is known that adding rays in the simplicial fan can give a crepant resolution. In the end of the paper we compute an example and explain that adding rays 
in the stacky hyperplane arrangement doesn't give a smooth hypertoric variety which means that it is not easy in general to give a crepant resolution in hyperkähler geometry.

Example 6.4. Let $\boldsymbol{\Sigma}=(N, \Sigma, \beta)$ be an extended stacky fan, where $N=\mathbb{Z}^{2}$, the simplicial fan $\Sigma$ is the fan of weighted projective plane $\mathbb{P}(1,1,2)$, and $\beta: \mathbb{Z}^{3} \rightarrow N$ is given by the vectors $\left\{b_{1}=(1,0), b_{2}=(0,1), b_{3}=(-1,-2),\right\}$, where $b_{1}, b_{2}, b_{3}$ are the generators of the rays in $\Sigma$. The generic element $\theta=(1) \in D G(\beta) \cong \mathbb{Z}$ determines the fan $\Sigma$. The stacky hyperplane arrangement $\mathcal{A}=(N, \beta, \theta)$ induces $\boldsymbol{\Sigma}$. The hypertoric DM stack is $\mathcal{M}(\mathcal{A})=T^{*}(\mathbb{P}(1,1,2))$. From Theorem 1.1,

$$
A_{\text {orb }}^{*}(\mathcal{M}(\mathcal{A})) \cong \frac{\mathbb{Q}\left[x_{1}, x_{2}, x_{3}, x_{4}\right]}{\left(x_{1}-x_{3}, x_{2}-2 x_{3}, x_{4}^{2}, x_{1} x_{2} x_{3}, x_{4} x_{2}, x_{4} x_{1} x_{3}\right)} \cong \frac{\mathbb{Q}\left[x_{3}, x_{4}\right]}{\left(x_{4}^{2}, x_{3}^{3}, x_{3} x_{4}\right)} .
$$

Let $b_{4}=(0,-1)$ and consider the new map $\beta^{\prime}: \mathbb{Z}^{4} \rightarrow N$ which is given by the vectors $\left\{b_{1}, b_{2}, b_{3}, b_{4}\right\}$. Choose generic element $\theta^{\prime}=(1,1) \in \mathbb{Z}^{2}=D G\left(\beta^{\prime}\right)$ and we get a new stacky hyperplane arrangement $\mathcal{A}^{\prime}=\left(N, \beta^{\prime}, \theta^{\prime}\right)$ which induces the extended stacky fan $\Sigma^{\prime}=\left(N, \Sigma, \beta^{\prime}\right)$. The hypertoric DM stack $\mathcal{M}\left(\mathcal{A}^{\prime}\right)$ is the stack corresponding to $\mathcal{A}^{\prime}$. From the definition of Box, $\left(\frac{1}{2} b_{1}+\frac{1}{2} b_{3}, \rho_{1}+\rho_{3}\right)$ is again a box element which determines a twisted sector. We compute that $A_{\text {orb }}^{*}\left(\mathcal{M}\left(\mathcal{A}^{\prime}\right)\right)$ is isomorphic to

$\frac{\mathbb{Q}\left[x_{1}, x_{2}, x_{3}, x_{4}, v\right]}{\left(x_{1}-x_{3}, x_{2}-2 x_{3}-x_{4}, x_{2} x_{4}, x_{1} x_{2} x_{3}, x_{1} x_{3} x_{4}, v^{2}, v x_{2}, v x_{4}\right)} \cong \frac{\mathbb{Q}\left[x_{3}, x_{4}, v\right]}{\left(x_{3} x_{4}+x_{4}^{2}, x_{3}^{3}, x_{3}^{2} x_{4}, v^{2}, v x_{3}, v x_{4}\right)}$. We check that $A_{\text {orb }}^{*}(\mathcal{M}(\mathcal{A}))$ is not isomorphic to the ring $A_{\text {orb }}^{*}\left(\mathcal{M}\left(\mathcal{A}^{\prime}\right)\right)$.

We give two comments here. First, if the crepant resolution conjecture is true, then $\mathcal{M}\left(\mathcal{A}^{\prime}\right)$ is not a hyperkähler resolution. On the other hand, the map $\beta$ is given by the matrix $B=$ $\left[\begin{array}{lll}1 & 0 & -1 \\ 0 & 1 & -2\end{array}\right]$ and the map $\beta^{\prime}$ given by $B^{\prime}=\left[\begin{array}{cccc}1 & 0 & -1 & 0 \\ 0 & 1 & -2 & -1\end{array}\right]$ It is easy to see that $B^{\prime}$ is not unimodular which means that $\mathcal{M}\left(\mathcal{A}^{\prime}\right)$ is not smooth, see [HS]. So adding columns in $B$ can't make it unimodular which means that adding rays in the stacky hyperplane arrangement can not give a hyperkähler resolution. Second, since $\mathcal{M}\left(\mathcal{A}^{\prime}\right)$ is still a hypertoric orbifold, we wish that $\mathcal{M}\left(\mathcal{A}^{\prime}\right)$ is a partial resolution and there is a morphism $\mathcal{M}\left(\mathcal{A}^{\prime}\right) \longrightarrow \mathcal{M}(\mathcal{A})$. But this is impossible because the two rings have different dimensions and it would violate the McKay correspondence statement if there exists a morphism $\mathcal{M}\left(\mathcal{A}^{\prime}\right) \longrightarrow \mathcal{M}(\mathcal{A})$, see [Ya1, [Ya2].

Question : Is there a combinatorial description of a hyperkähler resolution of hypertoric orbifolds?

\section{REFERENCES}

[AGV] D. Abramovich, T. Graber and A. Vistoli, Algebraic orbifold quantum product, in Orbifolds in mathematics and physics (Madison,WI,2001), 1-24, Contem. Math. 310, Amer. Math. Soc., 2002. math.AG/0112004.

[AH] A. A'Campo-Neuen and J. Hausen, Toric prevarieties and subtorus actions, Geometriae Dedicata 87, 35-64 (2001).

[BD] R. Bielawski and A. Dancer, The geometry and topology of toric hyperkähler manifolds, Comm. Anal. Geom. 8 (2000), 727-760.

[BCS] L. Borisov, L. Chen and G. Smith, The orbifold Chow ring of toric Deligne-Mumford stacks, $J$. Amer. Math. Soc. 18 (2005), no.1, 193-215, math.AG/0309229.

[BG] J. Bryan and T. Graber, The crepant resolution conjecture, math.AG/0610129. 
[BGP] J. Bryan, T. Graber and R. Pandharipande, The orbifold quantum cohomology of $\left[\mathbb{C}^{2} / \mathbb{Z}_{3}\right]$ and Hurwitz-Hodge integrals, math.AG/0510335.

[BJ] J. Bryan and Y. Jiang, The genus zero crepant resolution conjecture for the orbifold $\left[\mathbb{C}^{2} / \mathbb{Z}_{4}\right]$, preprint.

[CH] B. Chen and S. Hu, A deRham model for Chen-Ruan cohomology ring of abelian orbifolds, math.SG/0408265.

[CR1] W. Chen and Y. Ruan, A new cohomology theory for orbifolds, Comm. Math. Phys. 248 (2004), no. 1, 1-31, math.AG/0004129.

[CR2] W. Chen and Y. Ruan, Orbifold Gromov-Witten theory, in Orbifolds in mathematics and physics (Madison, WI, 2001), 25-85, Contem. Math. 310, Amer. Math. Soc., 2002. math.AG/0103156.

[Cox] D. Cox, The homogeneous coordinate ring of a toric variety, J. of Algebraic Geometry, 4 (1995), $17-50$.

[Cox2] D. Cox, Recent developments in toric geometry, in Algebraic Geometry (Santa Cruz, 1995), 389436, Proc. Symp. Pure Math. 62.2, Amer. Math. Soc., 1997.

[F] W. Fulton, Introduction to toric varieties, Annals of Mathematics Studies 131, Princeton University Press, Princeton, NJ, 1993.

[GH] R. Goldin and M. Harada, Orbifold cohomology of hypertoric varieties, math.DG/0607421.

[HM] A. Hattori and M. Masuda, Theory of multi-fans, Osaka J. Math. 40 (2003), 1-68.

[HS] T. Hausel and B. Sturmfels, Toric hyperkähler varieties, Documenta Mathematica, 7 (2002), 495534 .

[JKK] T. Jarvis, R. Kaufmann and T. Kimura, Stringy K-theory and the Chern character, math.AG/0502280

[Jiang] Y. Jiang, The orbifold cohomology ring of simplicial toric stack bundles, Illinois Journal of Mathematics, to appear, math.AG/0504563.

[JT] Y. Jiang and H.- H. Tseng, Note on orbifold Chow ring of semi-projective toric DM stacks, math.AG/0606322

[K] H. Konno, Cohomology rings of toric hyperkahler manifolds, Intern. J. of Math.(11) (1997), no.8, 1001-1026.

[Kro] P. Kronheimer, The construction of ALE spaces as hyper-Kähler quotients, J. Differential Geom. 29 (1989), no. 3, 665-683.

[KM] S. Keel and S. Mori, Quotients by groupoids, Ann. of Math. (2) 145 (1997), no.1, 193-213.

[L-MB] G. Laumon and L. Moret-Bailly, Champs algébriques. (French) [Algebraic stacks], Ergebnisse der Mathematik und ihrer Grenzgebiete. 3. Folge. 39. Springer-Verlag, Berlin, 2000.

[P] N. Proudfoot, Hyperkähler analogues of Kähler quotients, PhD thesis, UC Berkeley, spring 2004, math.AG/0405233

[PW] N. Proudfoot and B.Webster, Arithmetic and topology of hypertoric varieties, to appear in $J$. Algebraic Geom., math.AG/0411350.

[R] Y. Ruan, Cohomology ring of crepant resolutions of orbifolds, in Gromov-Witten theory of spin curves and orbifolds, 117-126, Contem. Math. 403, Amer. Math. Soc., 2006, math.AG/0108195.

[S] R. P. Stanley, Combinatorics and commutative algebra, 2nd ed., Birkhäuser Boston, 1996.

[V] A. Vistoli, Intersection theory on algebraic stacks and on their moduli spaces, Invent. Math., 97 (1989) 613-670.

[Ya1] T. Yasuda, Motivic integration over Deligne-Mumford stacks, math.AG/0312115.

[Ya2] T. Yasuda, Twisted jets, motivic measures and orbifold cohomology, Compos. Math. 140 (2004), no. $2,396-422$. 
Department of Mathematics, University of British Columbia, 1984 Mathematics Road, Vancouver, BC V6T 1Z2, Canada

E-mail address: jiangyf@math.ubc.ca

Department of Mathematics, University of British Columbia, 1984 Mathematics Road, Vancouver, BC V6T 1Z2, Canada

E-mail address: hhtseng@math.ubc.ca 